\title{
WEAK DECAY PROCESSES IN PRE-SUPERNOVA CORE EVOLUTION WITHIN THE GROSS THEORY
}

\author{
R. C. Ferreira ${ }^{1,5}$, A. J. Dimarco ${ }^{2}$, A. R. Samana ${ }^{2}$, and C. A. Barbero ${ }^{3,4}$ \\ ${ }^{1}$ Departamento de Estudos Básicos e Instrumentais, Universidade Estadual do Sudoeste da Bahia, BA, Brazil; roberto@uesb.edu.br \\ 2 Departamento de Ciências Exatas e Tecnológicas, Universidade Estadual de Santa Cruz, BA, Brazil \\ ${ }^{3}$ Departamento de Física, Universidad Nacional de La Plata, C.C. 67, 1900 La Plata, Argentina \\ ${ }^{4}$ Instituto de Física La Plata, CONICET, 1900 La Plata, Argentina \\ Received 2013 May 27; accepted 2013 December 15; published 2014 February 28
}

\begin{abstract}
The beta decay and electron capture rates are of fundamental importance in the evolution of massive stars in a pre-supernova core. The beta decay process gives its contribution by emitting electrons in the plasma of the stellar core, thereby increasing pressure, which in turn increases the temperature. From the other side, the electron capture removes free electrons from the plasma of the star core contributing to the reduction of pressure and temperature. In this work we calculate the beta decay and electron capture rates in stellar conditions for 63 nuclei of relevance in the pre-supernova stage, employing Gross Theory as the nuclear model. We use the abundances calculated with the Saha equations in the hypothesis of nuclear statistical equilibrium to evaluate the time derivative of the fraction of electrons. Our results are compared with other evaluations available in the literature. They have shown to be one order less or equal than the calculated within other models. Our results indicate that these differences may influence the evolution of the star in the later stages of pre-supernova.
\end{abstract}

Key words: atomic processes - nuclear reactions, nucleosynthesis, abundances - supernovae: general

Online-only material: color figures

\section{INTRODUCTION}

Research on the pre-supernova stage and also that related to supernovae explosions confronts many different obstacles that have lead to slow improvements in this area. First, a large obstacle is the small number of registered data available for supernovae events. In our galaxy 11 supernovae have been registered, and the frequency of explosions in a medium galaxy has recently been estimated in 1 event for every 50 years (Türler 2006). On the other hand, there are scarce observational data to compare and test the theoretical models.

Another obstacle is that, theoretically, more than one phenomena exists that can start gravitational collapse such as, photodissociation of iron and electron capture (EC). The study of these processes has its difficulties. Some of them are methods used to calculate the EC rates. In Arnet (1967), Bahcall (1964), and Colgate \& White (1966), simulations were performed for the real process without taking into account these rates for the involved nuclei, which lead to non-reliable results. Petterson \& Bahcall (1963) studied the influence of EC from the continuum in a high-density environment with densities from $10^{7}-10^{11} \mathrm{~g} \mathrm{~cm}^{-3}$. Several weak interaction processes were evaluated in Fowler \& Hoyle (1964) and Mazurek et al. (1974). Early in the 1980s, Fuller, Fowler, and Newman (hereafter FFN, Fuller et al. 1980) calculated the rates for positron emission, EC from continuum, and the loss of energy due to neutrinos for six reactions with nuclei in the region mass of $26 \leqslant A \leqslant 35$. Two years later, they extended the calculation to the mass region $26 \leqslant A \leqslant 60$ (Fuller et al. 1982a, 1982b). In the next three years, they showed simple expressions for the integral phase of positron and electron capture, and those related to the loss of energy due to neutrino emission (Fuller et al. 1985). In the 1990s, Kar et al. studied the beta decay (BD) and EC rates related to the most important reaction in the pre-supernova stage (Kar et al. 1994). In 1994, Aufderheide et al. (1994b, Auf) studied the influence of weak

\footnotetext{
5 Also at Programa de pós-graduação em física-PROFÍSICA, Universidade Estadual de Santa Cruz, BA, Brazil.
}

force in the phase stage before the gravitational collapse, taking into account the competition between the BD and EC rates. For the nuclear structure, calculations employed were the RPA and QRPA models, where the ground excited low energy, and excited high energy states were involved.

In the same decade, several authors (Alford et al. 1990; El-Kateb et al. 1994; Ronnquist et al. 1993; Vetterly et al. 1989; Williams et al. 1995) critiqued the systematic parameterization used by FFN (Fuller et al. 1980, 1982a, 1982b, 1985) and also by Auf (Aufderheide et al. 1994b). This fact was also highlighted by Aufderheide et al. (1996). Recently, Langanke \& MartínezPinedo (1999) and Dean et al. (1998), using a well-established theoretical basis, showed that the parameterization used by FFN and Auf in some important nuclei in the pre-supernova stage led to an overestimated distribution of Gamow-Teller strength relative to the experimental values. In 2000, Martínez-Pinedo et al. (2000) researched the competition between the BD and EC rates using the Shell Model (SM) for the nuclear structure calculations of 15 elements. In 2002, the importance of EC on the pre-supernova stage was reinforced in Sampaio et al. (2003). This research has thus evolved from the pioneering works to more modern research. However, theories about the pre-supernova stage and consequently supernovae are still very controversial, to the point that computer simulations have not successfully reproduced the explosion. Niu et al. (2011) pointed to the necessity of calculating more reliable rates, which represents an important issue that needs to be improved. One of the main insights of Auf's work was the realization that BDs were important during the early phase of collapse. Similar results were later found by the improved descriptions of weak interaction rates based on large-scale SM calculations. More importantly, the SM rates are in agreement with all the experimental charge exchange data available (Caurier et al. 1999; Cole et al. 2012). The SM-based rates (Langanke \& Martínez-Pinedo 2001) were used in pre-supernova stellar evolutionary calculations that, for the first time, included EC 
and BD processes (Heger et al. 2000, 2001) on the same footing.

In this context, here we provide our contribution. However, we note that we do not intend to give a final word on the problem, but simply to enrich discussions on the topic constraining some doubts arising from approaches that have been taken into account. Our main goal is to evaluate the $\mathrm{BD}$ and $\mathrm{EC}$ rates in the astrophysical environment for massive stars of $15 M_{\odot}-25 M_{\odot}$, using Gross Theory (GT) as the nuclear model and employing the most current experimental data. These rates will be useful to calculate the time derivative of the fraction of electrons and then describe their impact on the stellar trajectory of the presupernova and its consequences on the next stage of stellar evolution.

\subsection{Nuclear Models}

Nuclear theoretical models can be divided generically as (1) macroscopic models that describe the global nuclear properties (Takahashi \& Yamada 1969; Koyama et al. 1970; Kondoh et al. 1985; Tachibana et al. 1990; Nakata et al. 1997; Samana et al. 2008), where special attention is paid to the GT and (2) microscopic models, such as the SM or those based in the random phase approximation (RPA) calculations (Qian et al. 1997; Langanke 1999; Martínez-Pinedo 2001; Borzov \& Goriely 2000; Goriely \& Khan 2002), where the detailed nuclear structure of each specie is considered.

GT was first proposed by Takahashi \& Yamada (1969) nearly $40 \mathrm{yr}$ ago to describe global properties of allowed BD processes. It is essentially a parametric model, which attempted to combine single-particle and statistical arguments in a phenomenological way. In the following years, different versions of GT were developed, improved, and used for practical applications very frequently (Kondoh et al. 1985; Tachibana et al. 1990). This was essentially due to (1) their simplicity when compared with the difficult computational work involved in the implementation of the microscopic models, and (2) their capability to reproduce the available experimental data, and the possibility of being extrapolated later to unknown nuclei far from the $\beta$-stability line. In fact, as these theoretical approaches account systematically and fairly well for the properties of stable nuclei, they have been extensively applied to describe (1) the BD half-lives and other nuclear observables participating in the $r$-process, and (2) the properties of a great number of nuclei far from the line of $\beta$-stability that are involved in the nucleosynthesis.

GT has also been employed by Itoh \& Kohyama (1977) to calculate neutrino capture by ${ }^{37} \mathrm{Cl},{ }^{16} \mathrm{O},{ }^{20} \mathrm{Ne}$, and ${ }^{56} \mathrm{Fe}$ nuclei, which are used in the detection of solar neutrinos. Recently, it was employed to evaluate the BD half-lives, EC rates, and neutrino capture in nuclei with $A<70$, which are of major importance in pre-supernova collapse processes (Samana et al. 2008). That work analyzed the consequences of employing a more realistic estimation on the energetics of the Gamow-Teller resonance. This study led to a new trend for the adjustable parameter related to the energy spread of the Gamow-Teller resonance caused by the spin-dependent part of the nuclear force.

\subsection{Weak Decay Stellar Processes in Pre-supernova}

The importance of the electron fraction resides in the Chandrasekhar's mass given by

$$
M_{\mathrm{CH}}=5.80 Y_{e}^{2} M_{\odot},
$$

where $Y_{e}$ is the electron fraction defined as

$$
Y_{e}=\sum_{\alpha=1}^{r} \frac{x_{\alpha} Z_{\alpha}}{A_{\alpha}}
$$

The sum runs over $r$ nuclear species taking into account and $x_{\alpha}$, $Z_{\alpha}$, and $A_{\alpha}$ represent the abundance, charge, and mass of the $\alpha$ nuclear species, respectively. So, $M_{\mathrm{CH}}$ depends on the square of $Y_{e}$. The time derivative of $Y_{e}$ depends on the EC and BD rates, where the first one contributes to the missing electrons of stellar matter yielding a decrease in the pressure, while the second one brings electrons to stellar environment.

In particular, the time derivative of $Y_{e}$ due to EC and BD processes, according to Aufderheide et al. (1990), is

$$
\frac{d Y_{e}}{d t}=\sum_{Z, A} \frac{x(Z, A)}{A}\left[\lambda_{\mathrm{BD}}^{(Z, A)}-\lambda_{\mathrm{EC}}^{(Z, A)}\right],
$$

where the sum includes the nuclear species of major importance. Here, $\lambda_{\mathrm{BD}}^{(Z, A)}$ and $\lambda_{\mathrm{EC}}^{(Z, A)}$ are the $\mathrm{BD}$ and $\mathrm{EC}$ rates, respectively. Thus, the time derivative of $Y_{e}$ strongly depends on the rates and isotopic abundances. This fact shows us the importance of evaluating these quantities in a reliable way. From the pioneering work of FFN up to the present, it represents a major challenge in nuclear astrophysics. Our contribution to this topic is calculating the weak decay rates within GT. Relative to the abundances, due the fact that in the pre-supernova core all those reactions involving electromagnetic and nuclear interaction (but not the weak processes) are in equilibrium (NSE; Aufderheide et al. 1994b), we use the Saha equation to evaluate them for each nuclear species for a set of thermodynamics conditions $\left(\rho, T, Y_{e}\right)$. In this work, we adopted the abundances calculated in Dimarco et al. (2002).

\section{GROSS THEORY}

The BD and EC rates are calculated using Fermi's golden rule (we use natural units $m_{e}=\hbar=c=1$ ):

$$
\left\{\begin{array}{c}
\lambda_{\mathrm{BD}} \\
\lambda_{\mathrm{EC}}
\end{array}\right\}=\frac{G_{F}^{2}|M|^{2}}{2 \pi^{3}}\left\{\begin{array}{c}
f(Z+1, E) \\
g(Z, E)
\end{array}\right\},
$$

where $G_{F}=(3.034545 \pm 0.00006) \times 10^{-12}$ is the weak Fermi coupling constant, $|M|$ represents the nuclear matrix element (NME), and $f(Z, E)$ and $g(Z, E)$ are the Fermi integrals for the BD and EC processes, respectively. These integrals can be written as

$$
\begin{aligned}
\left\{\begin{array}{l}
f(Z, E) \\
g(Z, E)
\end{array}\right\}= & \int_{E_{1}}^{E_{2}}\left(E \pm 1 \mp E_{e}\right)^{2} E_{e} \\
& \times \sqrt{E_{e}^{2}-1} F\left(Z, E_{e}\right) d E_{e}
\end{aligned}
$$

where $F(Z, E)$ is the integrated Fermi function (Feenberg \& Trigg 1950; Rose 1961). The limits of integration are $E_{1}=1$ and $E_{2}=E+1$ for $\mathrm{BD}$, whereas that for $\mathrm{EC} E_{1}=\zeta$ and $E_{2}=\mathcal{E}_{F}+1$, with

$$
\zeta= \begin{cases}1 & \text { if } \quad E<0 \\ E+1 & \text { if } \quad E \geqslant 0\end{cases}
$$

Here $E=Q=M_{P}-M_{D}-1$ with $M_{P}$ and $M_{D}$ being the masses of parent and daughter nuclei, respectively, and $\mathcal{E}_{F}$ is the electron Fermi energy, which will be defined in Section 3. 
The NME are calculated using the GT originally proposed by Takahashi \& Yamada (1969) almost 40 yr ago. As in our previous work (Samana et al. 2008), we include the adjustable parameter related to the energy spread of the Gamow-Teller resonance caused by the spin-dependent part of the nuclear force. Gaussian type functions are assumed for the Fermi and Gamow-Teller resonances with energy, amplitude, and wide adjusted to recent experimental data. Essentially, the GT uses the NME obtained from the sum rule as it was explained in Kondoh et al. (1985).

In a first approximation, the total decay rates for $\mathrm{BD}$ and $\mathrm{EC}$ are written as

$$
\lambda_{\mathrm{BD}, \mathrm{EC}}=\lambda_{f}+\lambda_{\mathrm{gt}}+\lambda_{1}^{(0)}+\lambda_{1}^{(1)}+\lambda_{1}^{(2)} .
$$

Here, $\lambda_{f, \mathrm{gt}}$ are the allowed Fermi $(f)$ and Gamow-Teller $(\mathrm{gt})$ transitions, respectively, and $\lambda_{1}^{(0,1,2)}$ are the forbidden transitions of order 0,1 , and 2 . In many situations, the contributions of forbidden transitions are not relevant in comparison with allowed transitions, which leads to

$$
\lambda_{\mathrm{BD}, \mathrm{EC}} \simeq \lambda_{f}+\lambda_{\mathrm{gt}} .
$$

Using the sum rule for the NME, i.e.,

$$
\left|M_{\Omega}(E)\right|^{2}=\overline{\left|\left\langle\psi_{l}|\Omega| \psi_{0}\right\rangle\right|^{2}} \rho(E),
$$

the allowed rates for $\mathrm{BD}$ read

$$
\begin{aligned}
\lambda_{f, \mathrm{gt}}= & \frac{G_{F}^{2} g_{V, A}^{2}}{2 \pi^{3}} \sum_{l}\left|\left\langle\psi_{l}\left|\Omega_{f, \mathrm{gt}}\right| \psi_{0}\right\rangle\right|^{2} \\
& \times f\left(E_{0}-E_{l}\right),
\end{aligned}
$$

where the index 0 and $l$ represent the initial and final states, respectively, with the sum running over all the final states. $\psi_{0}$ and $\psi_{l}$ are the wave functions, $E_{0}$ and $E_{l}$ the energies, $g_{V}=1$ and $g_{A}=-1.2 g_{V} \cdot \Omega_{f} \equiv 1$ and $\Omega_{\mathrm{gt}} \equiv \sigma$ are the $f$ and gt nuclear operators, respectively. Then, using the sum rule, the weak decay rates are written as

$$
\begin{aligned}
& \lambda_{f}=\frac{G_{f}^{2} g_{V}^{2}}{2 \pi^{3}} \int_{-Q}^{0}\left|M_{f}(E)\right|^{2} f(-E) d E, \\
& \lambda_{\mathrm{gt}}=\frac{3 G_{F}^{2} g_{A}^{2}}{2 \pi^{3}} \int_{-Q}^{0}\left|M_{\mathrm{gt}}(E)\right|^{2} f(-E) d E,
\end{aligned}
$$

where $E$ is a continuum variable introducing to replace $E_{0}-E_{l}$, which leads to

$$
\begin{aligned}
\lambda_{\mathrm{BD}}= & \int_{-Q}^{0}\left[\mathcal{G}_{f}\left|M_{f}(E)\right|^{2}+3 \mathcal{G}_{\mathrm{gt}}\left|M_{\mathrm{GT}}(E)\right|^{2}\right] \\
& \times f(-E) d E,
\end{aligned}
$$

with $\mathcal{G}_{f, \mathrm{gt}}=\left(G_{F}^{2} g_{V, A}^{2} / 2 \pi^{3}\right)$.

The NME for BD within the GT are written as

$$
\left|M_{\Omega}(E)\right|^{2}=\int_{\epsilon_{0}(E)}^{\epsilon_{1}} D_{\Omega}(E, \epsilon) \frac{d N_{1}}{d \epsilon} W(E, \epsilon) d \epsilon,
$$

where $\epsilon_{1}$ is the energy of the highest occupied state and $\epsilon_{0}(E)=$ $\max \left(\epsilon_{\min }, \epsilon_{1}-Q-E\right)$ with $\epsilon_{\min }$ being the lowest single-particle energy of the parent nucleus. Here, $D_{\Omega}(E, \epsilon)$ is the singleparticle distribution normalized as $\int_{-\infty}^{+\infty} D_{\Omega}(E, \epsilon) d E=1$, because it represents the probability that a nucleon with singleparticle energy $\epsilon$ undergoes a $\beta$-transition. Pauli's principle is considered in the lower limit of the integral and in the term $W(E, \epsilon)$, which measures the probability of occupation of the final states (vacancy degree). Equation (11) is valid for the special case of a degree surface, where $W(E, \epsilon)=1$, because $\epsilon+E>\epsilon_{1}-Q$. In other cases, the term $W(E, \epsilon)$ vanishes because $\epsilon+E \leqslant \epsilon_{1}-Q$. Within this approximation, the NME reads

$$
\left|M_{\Omega}(E)\right|^{2}=\int_{\epsilon_{0}(E)}^{\epsilon_{1}} D_{\Omega}(E, \epsilon) \frac{d N_{1}}{d \epsilon} d \epsilon .
$$

The single-particle distribution $D_{\Omega}(E, \epsilon)$ was proposed in Takahashi \& Yamada (1969). To simplify, we do not consider dependence on $\epsilon$, which means that all the nucleons have the same probability to decay independently of their energy. The dependence on the parity of $N$ and $Z$ in the daughter nuclei is introduced through the pairing gap, $\Delta$, for single-particle levels adopted from Koyama et al. (1970). Successive improvements of the GT have used Gaussian-, exponential-, and Lorentziantype functions for $D_{\Omega}(E)$ (Takahashi \& Yamada 1969; Kondoh et al. 1985; Tachibana et al. 1990). Here, we use the Gaussiantype function because this one shows a reasonable agreement with the data, as it was noted in Samana et al. (2008). Similar results are obtained with Lorentzian strength functions. The Gaussian-type function $D_{\Omega}(E)$ is

$$
D_{\Omega}(E)=\frac{1}{\sqrt{2 \pi} \sigma_{\Omega}} e^{\frac{-\left(E-E_{\Omega}\right)^{2}}{2 \sigma_{\Omega}^{2}}} .
$$

Here, $E_{\Omega}$ is the resonance energy and $\sigma_{\Omega}$ the standard deviation. Following Takahashi \& Yamada (1969), we assume the nuclei as an uniform charged sphere with radius $1.2 A^{1 / 3} \mathrm{fm}$, which leads to

$$
\begin{gathered}
E_{f}=E_{c}= \pm\left(1.44 Z_{1} A^{-\frac{1}{3}}-0.7825\right) \mathrm{MeV} \\
\sigma_{f}=\sigma_{c}=0.157 Z_{1} A^{-\frac{1}{3}}
\end{gathered}
$$

where $Z_{1}$ is the proton number of the daughter (parent) nuclei for $\beta^{+}\left(\beta^{-}\right)$decay. For the Gamow-Teller resonance, we used the estimate proposed by Nakayama et al. (1982), also employed in Samana et al. (2008),

$$
E_{\mathrm{gt}}=E_{f}+\delta
$$

with $\delta$ defined as

$$
\delta=26 A^{-\frac{1}{3}}-\frac{18.5(N-Z)}{A} \mathrm{MeV} .
$$

The standard deviation is adjusted with the factor $\sigma_{N}$, which has the correction in the energies coming from the nuclear spin dependent forces. Then

$$
\sigma_{\mathrm{gt}}=\sqrt{\sigma_{C}^{2}+\sigma_{N}^{2}}
$$

The adopted values for $\sigma_{N}$ were those of Samana et al. (2008) obtained in a $\chi^{2}$-minimization procedure with nuclei $A<70$.

Following the original version of the GT, the Fermi gas model was used to estimate $d N_{1} / d \epsilon$, as

$$
\frac{d N_{1}}{d \epsilon}=N_{1}\left[1-\left(1-\frac{Q+E}{\epsilon_{F}}\right)^{\frac{3}{2}}\right]
$$


Here, the factor $N_{1}$ is the number of neutrons of the parent nuclei for $\beta^{-}$, whereas for EC $N_{1}=Z . \epsilon_{F}$ is the nucleon Fermi energy given by

$$
\epsilon_{F}=\frac{76.52}{\frac{M_{n}^{*}}{M_{n}}} \frac{1}{r_{o}^{2}}\left(\frac{N_{1}}{A}\right)^{\frac{2}{3}} \mathrm{MeV},
$$

with $M_{n}^{*}$ and $M_{n}$ being the nucleon effective and bare masses, respectively, and $A$ is the mass number. We used the relations $r_{o}=1.25\left(1+0.65 A^{-2 / 3}\right)$ and $M_{n}^{*} / M_{n}=0.6+0.4 A^{-1 / 3}$.

The same procedure can be directly repeated for EC rates. Thus, replacing the values of $\left(d N_{1} / d \epsilon\right)$ and using the notation $f(-E)$ and $g(-E)$ for the Fermi functions, the total decay rates finally read

$$
\begin{aligned}
\left\{\begin{array}{c}
\lambda_{\mathrm{BD}} \\
\lambda_{\mathrm{EC}}
\end{array}\right\}= & \int_{-Q}^{\left\{\begin{array}{c}
0 \\
\epsilon_{F}
\end{array}\right\}}\left[\mathcal{G}_{f} D_{f}(E)+3 \mathcal{G}_{\mathrm{gt}} D_{\mathrm{gt}}(E)\right] \\
& \times\left\{\begin{array}{c}
N \\
Z
\end{array}\right\}\left[1-\left(1-\frac{Q+E}{\epsilon_{F}}\right)^{\frac{3}{2}}\right]\left\{\begin{array}{l}
f(-E) \\
g(-E)
\end{array}\right\} d E .
\end{aligned}
$$

\section{WEAK DECAY RATES IN STELLAR CONDITIONS}

In this section, we introduce the effects of density and temperature present in stellar environments over BD and EC decay rates.

In the original GT from Takahashi \& Yamada (1969), the nuclear disintegration is from the parent ground state to the daughter one. When the temperatures are of the order of $10^{9} \mathrm{~K}$, the probability of finding the parent nuclei in the ground state can be estimated as

$$
P(0)=\frac{\left(2 J_{0}+1\right) e^{\left(\frac{-E_{0}}{K_{B} T}\right)}}{G(A, Z, T)},
$$

where $J_{0}$ is the spin of ground state, $E_{0}$ is the ground state energy for the parent nuclei and considered as zero for every nuclei, $K_{B}$ is the Boltzmann constant, $T$ is the temperature in Kelvin, and $G(A, Z, T)$ is the nuclear partition function, where we adopt the values calculated in Dimarco et al. (2002). We use the values of temperature and density of the parameterization performed by Auf (Aufderheide et al. 1994b), valid for massive stars with $15 M_{\odot}$ to $25 M_{\odot}$

$$
\begin{aligned}
\log _{10} \rho\left(Y_{e}\right) & =603-3642 Y_{e}+7439 Y_{e}^{2}-5075 Y_{e}^{3}, \\
T_{9}\left(Y_{e}\right) & =1212-7571 Y_{e}+15831 Y_{e}^{2}-11047 Y_{e}^{3} .
\end{aligned}
$$

Here $T_{9}$ is the temperature in $10^{9} \mathrm{~K}$ and $\rho$ is the density in $\mathrm{g} \mathrm{cm}^{-3}$. This parameterization was generated from the KEPLER code (Weaver et al. 1978), using the EC rates from FFN (Fuller et al. 1982a) and the BD ones from a statistical model used by Mazurek et al. (1974). This is not an updated parameterization, but it gives the possibility to compare our results with those of Auf (Aufderheide et al. 1994b) and other authors that use the same density and temperature values.

Another modification to perform in stellar conditions is because of the behavior of free electrons in the pre-supernova core. In the previous section, we showed that the Coulombian interaction of the electron with the parent nuclei is considered using the Fermi function $F(Z, E)$. However, we must emphasize that in the core of the pre-supernova we have a degenerate electron sea. At zero and lower temperatures, the energy of the last electron level occupied corresponds to $\mathcal{E}_{F}$. In this case, the relativistic kinetic Fermi energy for electrons proposed in Egawa et al. (1975) was $\mathcal{E}_{F}=\left(P_{F}^{2}+1\right)^{1 / 2}-1$ with

$$
P_{F}=\left(3 \pi^{2} N_{A} \rho Y_{e}\right)^{1 / 3},
$$

where $\rho$ is the mass density and $N_{A}$ is Avogadro's number. However, at finite $T$, the electron energy distribution for EC is the Fermi-Dirac distribution

$$
f_{\mathrm{EC}}\left(E_{e}\right)=\frac{1}{1+e^{\left(\frac{E_{e}-\mu_{e}}{K_{B} T}\right)},}
$$

and for BD we have

$$
f_{\mathrm{DB}}\left(E_{e}\right)=\frac{1}{1+e^{\left(\frac{\mu_{e}-E_{e}}{K_{B} T}\right)}} .
$$

Here $\mu_{e}$ is the chemical potential in $\mathrm{MeV}$. We used for $\mu_{e}$ the approximation given in Aufderheide et al. (1990)

$$
\begin{aligned}
\mu_{e}= & 1.11\left(\rho_{7} Y_{e}\right)^{1 / 3} \\
\times & {\left[1+\left(\frac{\pi}{1.11}\right)^{2} \frac{T^{2}}{\left(\rho_{7} Y_{e}\right)^{2 / 3}}\right]^{-1 / 3} \mathrm{MeV}, }
\end{aligned}
$$

where $\rho_{7}$ is the density in $10^{7} \mathrm{~g} \mathrm{~cm}^{-3}$ and $T$ is the temperature in $\mathrm{MeV}$. Thus, for $\mathcal{E}_{F}$ as a function of temperature, we use $\mathcal{E}_{F}=\mu_{e}$.

Multiplying the Fermi-Dirac distribution times the Fermi function $F\left(Z, E_{e}\right)$, the Equation (4) for BD in stellar conditions goes to

$$
\begin{aligned}
f\left(-E, \rho, T, Y_{e}\right)= & \int_{1}^{-E+1}\left(-E+1-E_{e}\right)^{2} E_{e} \\
& \times \sqrt{E_{e}^{2}-1} F\left(Z+1, E_{e}\right) f_{\mathrm{DB}}\left(E_{e}\right) d E_{e} .
\end{aligned}
$$

Introducing all these considerations in Equation (21), the BD rate in stellar conditions is

$$
\begin{aligned}
\lambda_{\mathrm{BD}}( & \left.E, \rho, T, Y_{e}\right)=P(0) \int_{-Q}^{0}\left[\mathcal{G}_{f} D_{f}(E)\right. \\
& \left.+3 \mathcal{G}_{\mathrm{gt}} D_{\mathrm{gt}}(E)\right] \\
& \times N\left[1-\left(1-\frac{Q+E}{\epsilon_{F}}\right)^{\frac{3}{2}}\right] f\left(-E, \rho, T, Y_{e}\right) d E .
\end{aligned}
$$

Similarly, for EC the integral of Fermi function reads

$$
\begin{aligned}
g\left(-E, \rho, T, Y_{e}\right)= & \int_{\zeta}^{\mathcal{E}_{F}+1}\left(-E-1+E_{e}\right)^{2} E_{e} \\
& \times \sqrt{E_{e}^{2}-1} F\left(Z, E_{e}\right) f_{\mathrm{EC}}\left(E_{e}\right) d E_{e}
\end{aligned}
$$

In the same way as for $\mathrm{BD}$, we can finally write

$$
\begin{aligned}
\lambda_{\mathrm{EC}}( & \left.E, \rho, T, Y_{e}\right)=P(0) \int_{-Q}^{\mathcal{E}_{F}}\left[\mathcal{G}_{f} D_{f}(E)\right. \\
& \left.+3 \mathcal{G}_{\mathrm{gt}} D_{\mathrm{gt}}(E)\right] \\
& \times Z\left[1-\left(1-\frac{Q+E}{\epsilon_{F}}\right)^{\frac{3}{2}}\right] g\left(-E, \rho, T, Y_{e}\right) d E .
\end{aligned}
$$




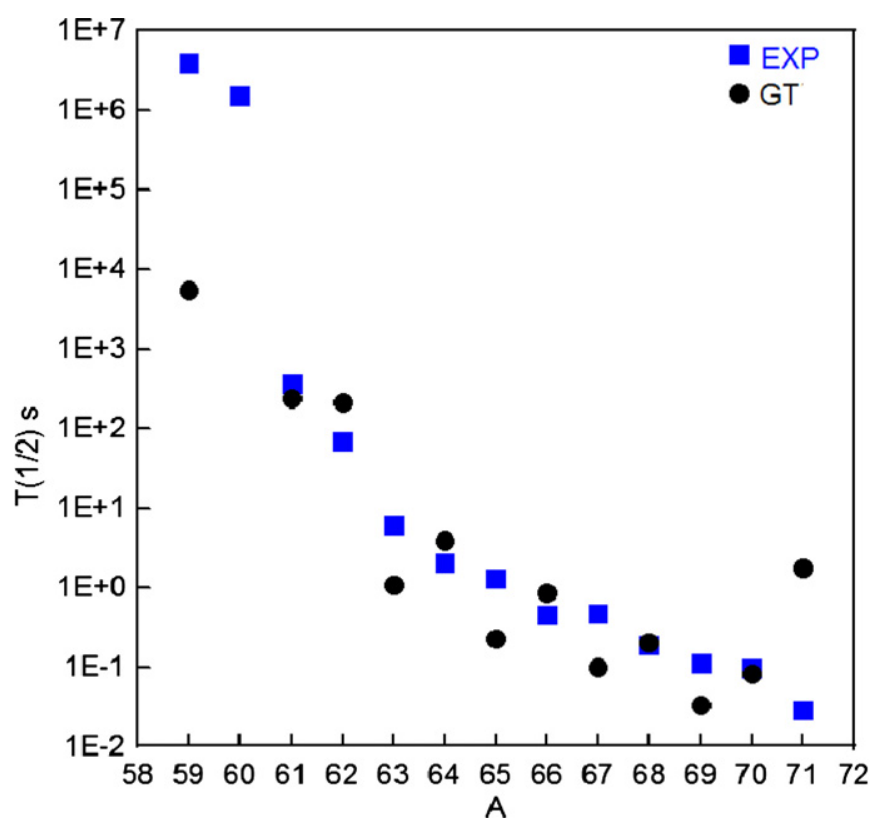

Figure 1. Beta decay lifetimes for the iron isotopes (experimental data from Letter of Nuclide 2013).

(A color version of this figure is available in the online journal.)

\section{RESULTS AND DISCUSSION}

In our calculation we have included 63 nuclear species with major abundance in the pre-supernova phase. The used nuclei were ${ }^{49,50} \mathrm{Ca},{ }^{50,51} \mathrm{Sc}$, Ti with $51 \leqslant A \leqslant 53$, V with $50 \leqslant A \leqslant$ 54 , Cr with $51 \leqslant A \leqslant 56$, Mn with $55 \leqslant A \leqslant 60$, Fe with $55 \leqslant A \leqslant 63$, Co with $55 \leqslant A \leqslant 63$, Ni with $56 \leqslant A \leqslant 68$, $\mathrm{Cu}$ with $60 \leqslant A \leqslant 63$, and $\mathrm{Zn}$ with $60 \leqslant A \leqslant 63$. As we mentioned previously, this version of the GT model treats the nuclear disintegration starting from the ground state in the parent nucleus and, by this reason, 34 of those nuclei exhibit values with $Q<0$ for $\mathrm{BD}$ and the $\mathrm{BD}$ rates cannot be calculated.

Our calculations reproduce the available experimental data on charge-exchange reactions (see Figures 3 and 4 in Samana et al. 2008). Without temperature dependence, the results we obtained from Equation (21) show the following.

1. For BD, our model successfully represents the experimental data. For example, this behavior is shown in Figure 1 for the iron family. We note that, for the sake of completeness, we have tested that the present GT model describes the experimental data, as well as other more evolved versions of the GT (Tachibana et al. 1990) and showing minor differences (almost 5\%) in the calculated rates.

2. The rates for EC are very sensitive to the chemical potential $\mu_{e}$, showing an increase for higher values of $Y_{e}$. In addition, $\mu_{e}$ is very sensitive to $Y_{e}$ : we observed that for $0.40 \leqslant$ $Y_{e} \leqslant 0.50$ (which gives density values on the order of $10^{7}-10^{10} \mathrm{~g} \mathrm{~cm}^{-3}$ ) we find values $1.17 \leqslant \mu_{e} \leqslant 10.37 \mathrm{MeV}$. This is because we have a high increase of degeneracy pressure, which is proportional to the density.

When the temperature dependence is included, the decay rates obtained from Equations (29) and (31) exhibit the behavior as expected from Aufderheide et al. (1994b), i.e., there is an increase of the EC rates when the electron fraction value is reduced, whereas for $\mathrm{BD}$ the opposite happens because the rates decrease when the electron fraction is diminished. This is shown in Figures 2 and 3, where we plot the rates for EC of cobalt,
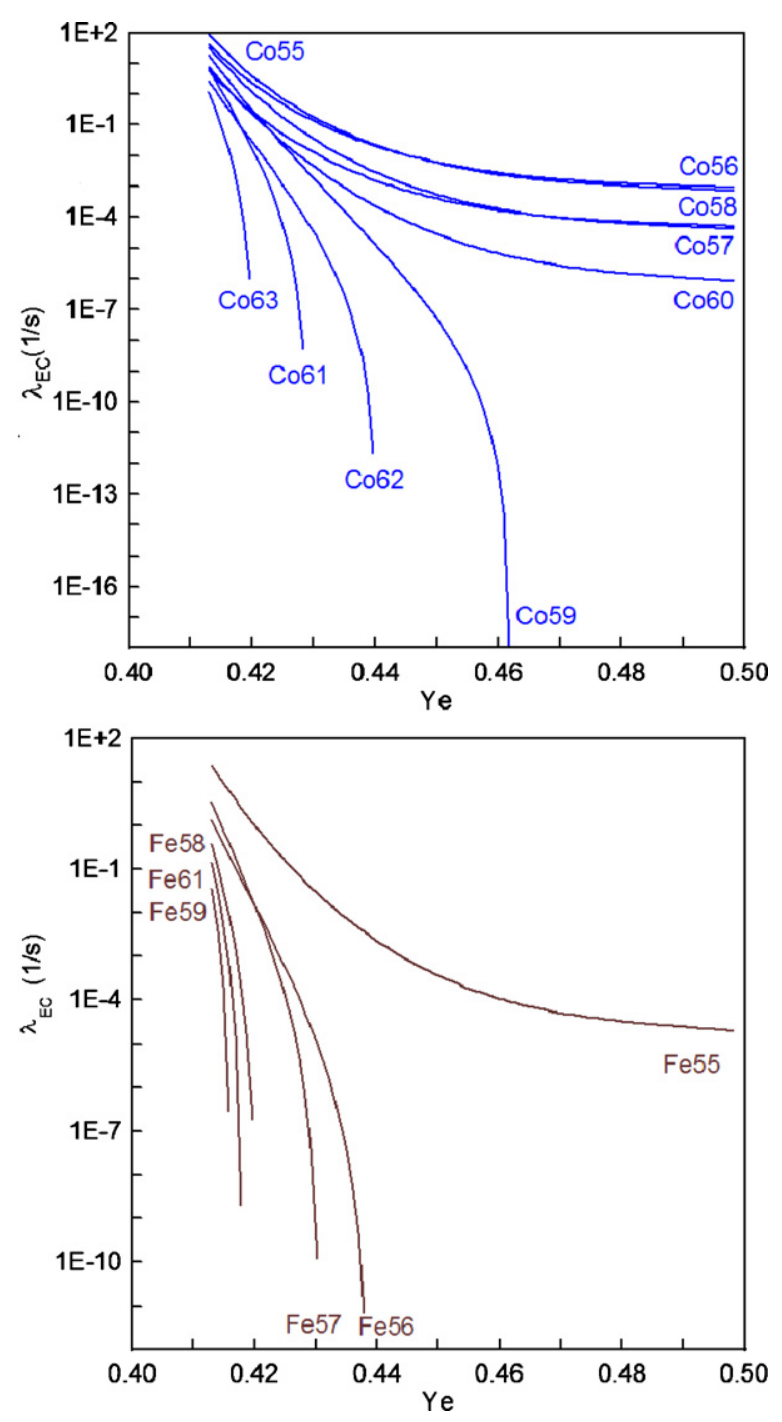

Figure 2. Electron capture rates for cobalt and iron families. (A color version of this figure is available in the online journal.)

iron, manganese, and nickel. In Figures 4 and 5 the results for $\mathrm{BD}$ rates of the same families are sketched.

In order to estimate the effects of the decay rates on some observable of astrophysical interest, we will calculate the temporal derivative of the electron fraction, $\dot{Y}_{e}$, given by Equation (2). As described previously, for this evaluation we use the isotopic abundances calculated in Dimarco et al. (2002). It is important to mention here that several authors (Alford et al. 1990; El-Kateb et al. 1994; Ronnquist et al. 1993; Vetterly et al. 1989; Williams et al. 1995) discussed the systematic method used by FFN and Auf on the parameterization of the nuclear matrix element of some nuclei, as pointed out recently in Aufderheide et al. (1996). With stronger theoretical fundament, Langanke \& Martínez-Pinedo (1999) and Dean et al. (1998) have shown that the parameterization used for some nuclei, considered as important in the pre-supernova stage, exhibit overestimated theoretical decay rates for the Gamow-Teller distribution when compared with the experimental data. In order to determine whether our model overestimated the decay rates, we compared our results for some nuclear elements with those obtained by FFN (Fuller et al. 1982a), Auf (Aufderheide et al. 1994b), and Martínez-Pinedo et al. (2000) SM calculations. The comparison for the EC rates are presented in Tables 1-7, and 
Table 1

Electron Capture for the Eight More Relevant Nuclei at $Y_{e}=0.485$

\begin{tabular}{|c|c|c|c|c|c|c|}
\hline Nucleus & $\begin{array}{c}\lambda_{\mathrm{EC}}(\mathrm{GT}) \\
\left(\mathrm{s}^{-1}\right)\end{array}$ & $\begin{array}{c}\lambda_{\mathrm{EC}}(\mathrm{SM}) \\
\left(\mathrm{s}^{-1}\right)\end{array}$ & $\begin{array}{c}\lambda_{\mathrm{EC}}(\text { Auf }) \\
\left(\mathrm{s}^{-1}\right)\end{array}$ & $\begin{array}{c}\lambda_{\mathrm{EC}}(\mathrm{FFN}) \\
\left(\mathrm{s}^{-1}\right)\end{array}$ & $\begin{array}{c}\left|\dot{Y}_{e \mathrm{EC}}(\mathrm{GT})\right| \\
\left(\mathrm{s}^{-1}\right)\end{array}$ & $\begin{array}{c}\mid \dot{Y}_{e \mathrm{EC}}(\text { Auf }) \mid \\
\left(\mathrm{s}^{-1}\right)\end{array}$ \\
\hline$\overline{{ }^{57} \mathrm{Ni}}$ & $3.62 \times 10^{-6}$ & $\ldots$ & $1.34 \times 10^{-2}$ & $\ldots$ & $3.42 \times 10^{-6}$ & $5.30 \times 10^{-6}$ \\
\hline${ }^{56} \mathrm{Ni}$ & $1.25 \times 10^{-3}$ & $6.30 \times 10^{-3}$ & $8.55 \times 10^{-3}$ & $8.60 \times 10^{-3}$ & $2.97 \times 10^{-6}$ & $2.09 \times 10^{-5}$ \\
\hline${ }^{55} \mathrm{Co}$ & $9.22 \times 10^{-4}$ & $2.20 \times 10^{-3}$ & $5.13 \times 10^{-2}$ & $8.40 \times 10^{-2}$ & $1.95 \times 10^{-6}$ & $4.80 \times 10^{-5}$ \\
\hline${ }^{56} \mathrm{Co}$ & $1.13 \times 10^{-3}$ & $1.00 \times 10^{-2}$ & $3.42 \times 10^{-2}$ & $\ldots$ & $2.54 \times 10^{-7}$ & $8.34 \times 10^{-7}$ \\
\hline${ }^{58} \mathrm{Ni}$ & $3.18 \times 10^{-6}$ & $\ldots$ & $3.86 \times 10^{-4}$ & $\ldots$ & $3.33 \times 10^{-7}$ & $1.66 \times 10^{-6}$ \\
\hline${ }^{57} \mathrm{Co}$ & $5.46 \times 10^{-5}$ & $\ldots$ & $2.36 \times 10^{-3}$ & $\ldots$ & $2.02 \times 10^{-7}$ & $1.11 \times 10^{-7}$ \\
\hline${ }^{59} \mathrm{Ni}$ & $1.49 \times 10^{-4}$ & $\ldots$ & $3.06 \times 10^{-3}$ & $\ldots$ & $1.70 \times 10^{-8}$ & $2.66 \times 10^{-8}$ \\
\hline \multirow[t]{2}{*}{${ }^{55} \mathrm{Fe}$} & $2.73 \times 10^{-5}$ & $\ldots$ & $1.06 \times 10^{-3}$ & $\ldots$ & $1.43 \times 10^{-8}$ & $5.72 \times 10^{-8}$ \\
\hline & & $\rho_{7}=4.32$ & $T_{9}=3.26$ & & $\begin{array}{c}\dot{Y}_{e \mathrm{EC}}(\mathrm{GT}) \\
-8.69 \times 10^{-6}\end{array}$ & $\begin{array}{c}\dot{Y}_{e \mathrm{EC}}(\text { Auf }) \\
-7.95 \times 10^{-5}\end{array}$ \\
\hline
\end{tabular}
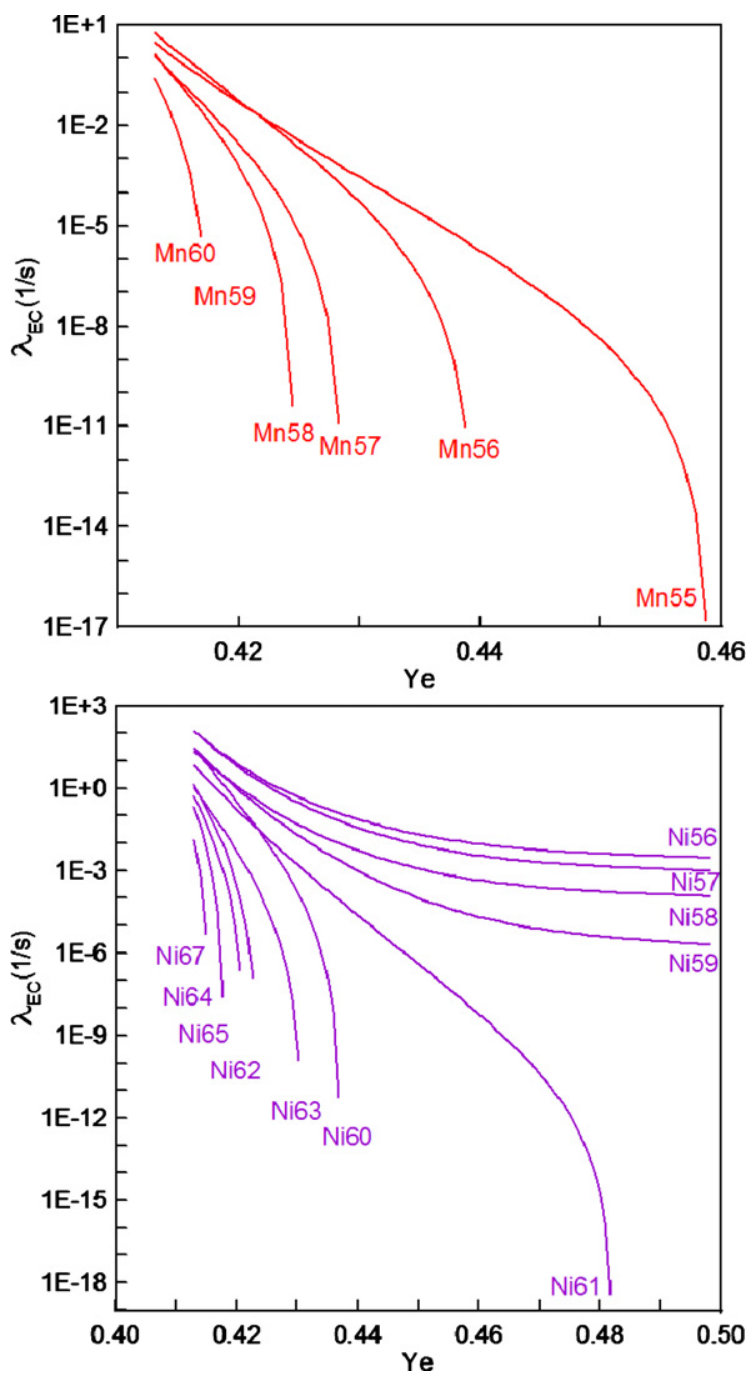

Figure 3. Electron capture rates for manganese and nickel families. (A color version of this figure is available in the online journal.)

those for BD in Tables 8-13. There we list the values of

$$
\dot{Y}_{e}=\dot{Y}_{e \mathrm{BD}}+\dot{Y}_{e \mathrm{EC}},
$$

being

$$
\begin{aligned}
& \dot{Y}_{e \mathrm{EC}}=\frac{-x(Z, A)}{A} \lambda_{\mathrm{EC}}^{(Z, A)}, \\
& \dot{Y}_{e \mathrm{BD}}=\frac{x(Z, A)}{A} \lambda_{\mathrm{BD}}^{(Z, A)},
\end{aligned}
$$
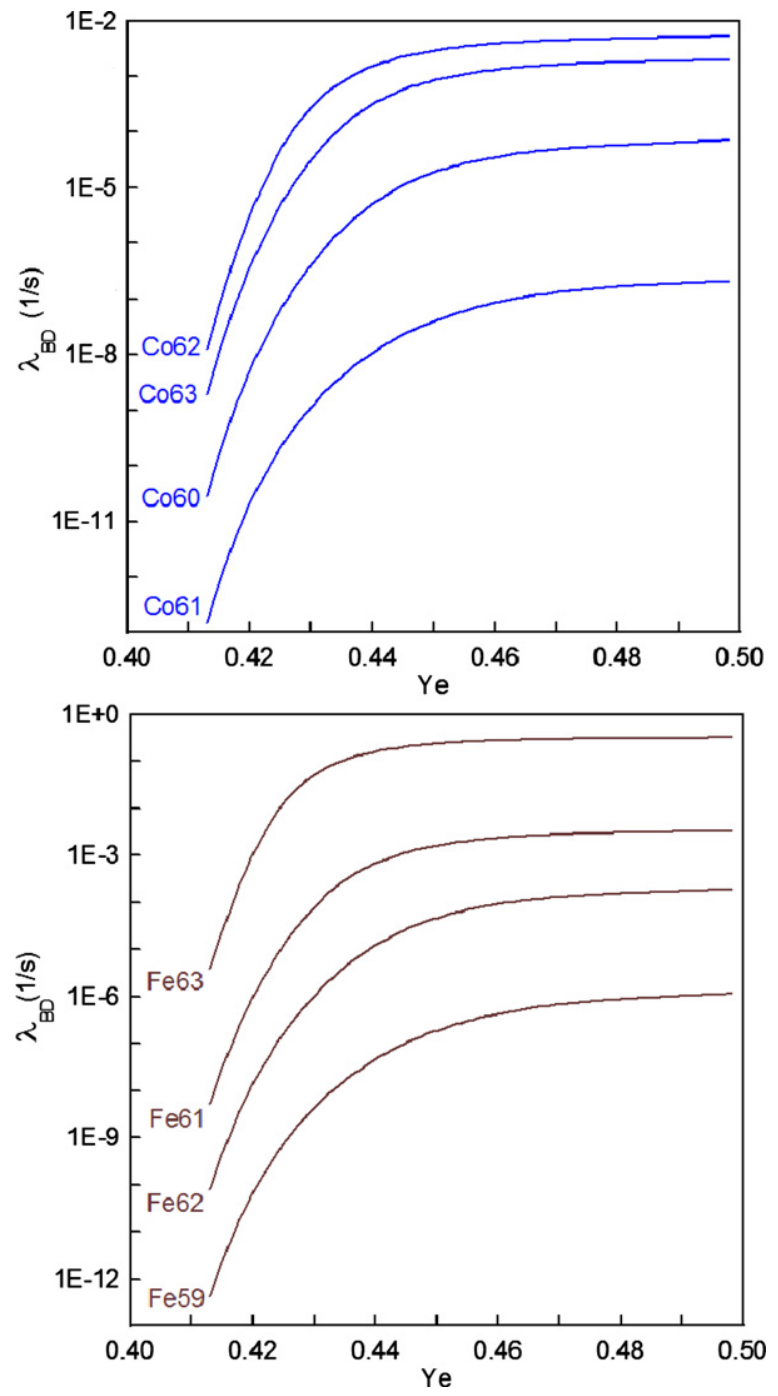

Figure 4. Beta decay rates for cobalt and iron families.

(A color version of this figure is available in the online journal.)

for eight specific values of $Y_{e}$. They are all ordered according to the decreasing value of $\dot{Y}_{e}$ calculated within the GT. The sum of the $\dot{Y}_{e}$ values for the GT presented at the end of each table with the symbol $\dot{Y}_{e}^{\text {tot }}(\mathrm{GT})$ was performed over all those nuclei, which show a curve of $\dot{Y}_{e}$ passing through this point. The corresponding values calculated by Auf, symbolized as $\dot{Y}_{e}^{\text {tot }}$ (Auf), represent the summation of the 20 more important 
Table 2

Electron Capture for the 12 More Relevant Nuclei at $Y_{e}=0.47$

\begin{tabular}{|c|c|c|c|c|c|c|}
\hline Nucleus & $\begin{array}{c}\lambda_{\mathrm{EC}}(\mathrm{GT}) \\
\left(\mathrm{s}^{-1}\right)\end{array}$ & $\begin{array}{c}\lambda_{\mathrm{EC}}(\mathrm{SM}) \\
\left(\mathrm{s}^{-1}\right)\end{array}$ & $\begin{array}{c}\lambda_{\mathrm{EC}} \text { (Auf) } \\
\left(\mathrm{s}^{-1}\right)\end{array}$ & $\begin{array}{c}\lambda_{\mathrm{EC}}(\mathrm{FFN}) \\
\left(\mathrm{s}^{-1}\right)\end{array}$ & $\begin{array}{c}\left|\dot{Y}_{e \mathrm{EC}}(\mathrm{GT})\right| \\
\left(\mathrm{s}^{-1}\right)\end{array}$ & $\begin{array}{c}\mid \dot{Y}_{e \mathrm{EC}}(\text { Auf }) \mid \\
\left(\mathrm{s}^{-1}\right)\end{array}$ \\
\hline$\overline{{ }^{57} \mathrm{Ni}}$ & $5.94 \times 10^{-3}$ & 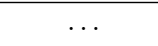 & $1.94 \times 10^{-2}$ & $9.93 \times 10^{-3}$ & $2.45 \times 10^{-7}$ & $5.84 \times 10^{-8}$ \\
\hline${ }^{56} \mathrm{Co}$ & $1.68 \times 10^{-3}$ & $1.70 \times 10^{-3}$ & $7.40 \times 10^{-2}$ & $6.91 \times 10^{-2}$ & $1.89 \times 10^{-7}$ & $7.35 \times 10^{-7}$ \\
\hline${ }^{55} \mathrm{Co}$ & $1.68 \times 10^{-3}$ & $\cdots$ & $1.41 \times 10^{-1}$ & $1.36 \times 10^{-1}$ & $1.73 \times 10^{-7}$ & $1.37 \times 10^{-6}$ \\
\hline${ }^{57} \mathrm{Co}$ & $9.35 \times 10^{-5}$ & $1.50 \times 10^{-4}$ & $3.50 \times 10^{-3}$ & $1.89 \times 10^{-3}$ & $1.25 \times 10^{-7}$ & $2.18 \times 10^{-6}$ \\
\hline${ }^{55} \mathrm{Fe}$ & $5.01 \times 10^{-5}$ & $2.70 \times 10^{-4}$ & $3.80 \times 10^{-3}$ & $5.80 \times 10^{-3}$ & $1.14 \times 10^{-7}$ & $1.52 \times 10^{-6}$ \\
\hline${ }^{59} \mathrm{Ni}$ & $2.41 \times 10^{-4}$ & $4.90 \times 10^{-4}$ & $4.37 \times 10^{-3}$ & $4.31 \times 10^{-3}$ & $8.69 \times 10^{-8}$ & $3.80 \times 10^{-7}$ \\
\hline${ }^{56} \mathrm{Ni}$ & $1.98 \times 10^{-3}$ & & $1.60 \times 10^{-2}$ & $1.30 \times 10^{-2}$ & $2.66 \times 10^{-8}$ & $7.76 \times 10^{-9}$ \\
\hline${ }^{58} \mathrm{Ni}$ & $7.42 \times 10^{-6}$ & $8.40 \times 10^{-5}$ & $6.36 \times 10^{-4}$ & $3.72 \times 10^{-4}$ & $2.45 \times 10^{-8}$ & $5.97 \times 10^{-7}$ \\
\hline${ }^{51} \mathrm{Cr}$ & $1.44 \times 10^{-4}$ & & $2.81 \times 10^{-3}$ & $1.31 \times 10^{-7}$ & $3.61 \times 10^{-9}$ & $1.97 \times 10^{-8}$ \\
\hline${ }^{58} \mathrm{Co}$ & $9.73 \times 10^{-5}$ & $5.00 \times 10^{-4}$ & $4.04 \times 10^{-2}$ & $3.68 \times 10^{-2}$ & $2.60 \times 10^{-9}$ & $2.35 \times 10^{-8}$ \\
\hline${ }^{61} \mathrm{Cu}$ & $4.96 \times 10^{-4}$ & $\ldots$ & $3.93 \times 10^{-1}$ & $\ldots$ & $1.83 \times 10^{-9}$ & $3.22 \times 10^{-7}$ \\
\hline \multirow[t]{2}{*}{${ }^{60} \mathrm{Cu}$} & $4.07 \times 10^{-3}$ & $\cdots$ & $8.39 \times 10^{-1}$ & $1.20 \times 10^{-1}$ & $3.30 \times 10^{-10}$ & $4.30 \times 10^{-9}$ \\
\hline & & 5.86 & $=3.4$ & & $\begin{array}{c}\dot{Y}_{e \mathrm{EC}}(\mathrm{GT}) \\
-9.96 \times 10^{-7}\end{array}$ & $\begin{array}{c}\dot{Y}_{e \mathrm{EC}}(\text { Auf }) \\
-8.07 \times 10^{-6}\end{array}$ \\
\hline
\end{tabular}
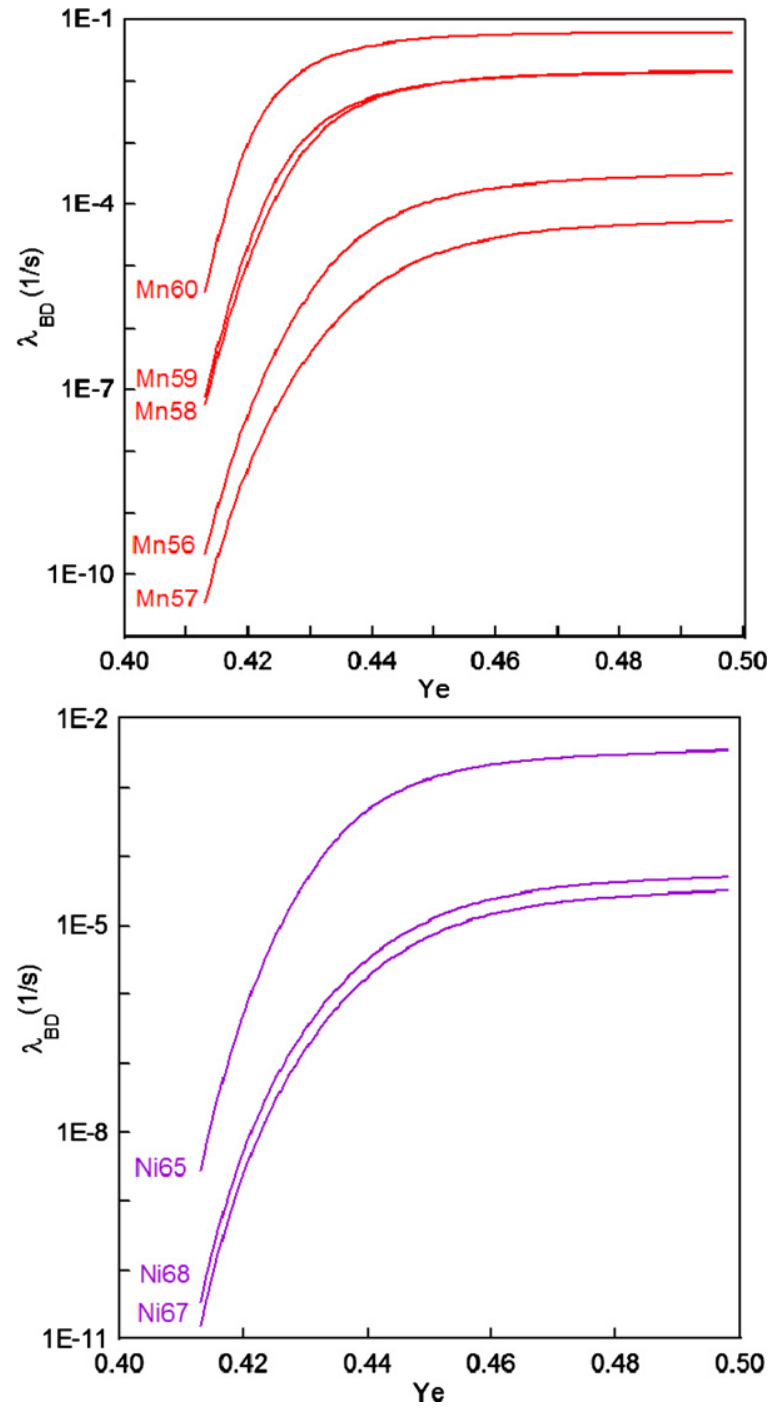

Figure 5. Beta decay rates for manganese and nickel families. (A color version of this figure is available in the online journal.)

nuclei in the specified point of the stellar trajectory, considering those species with higher values of $\dot{Y}_{e}$.

We plot the results for EC in Figures 6 and 7. In Figure 6, following Aufderheide et al. (1994b) and Martínez-Pinedo
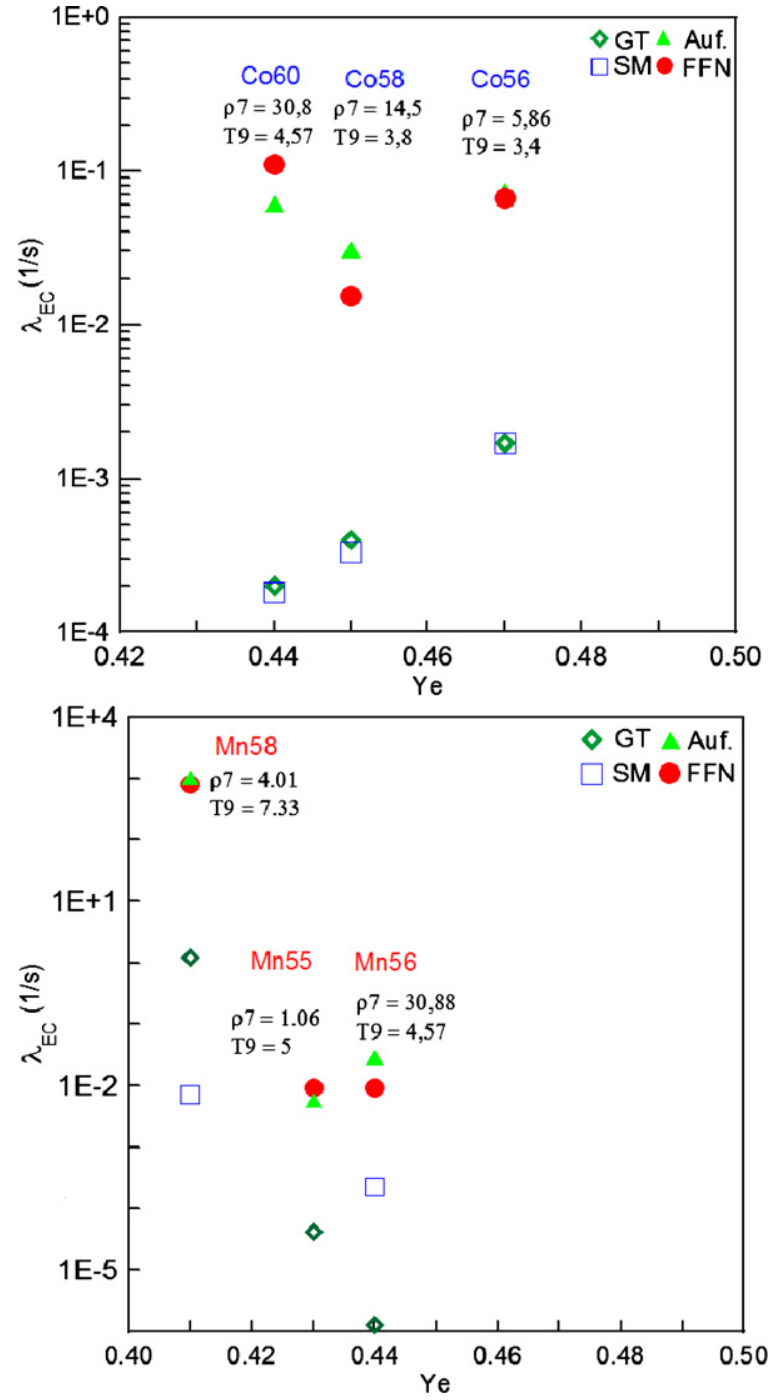

Figure 6. Electron capture rates for ${ }^{56} \mathrm{Co},{ }^{58} \mathrm{Co},{ }^{60} \mathrm{Co},{ }^{55} \mathrm{Mn},{ }^{56} \mathrm{Mn}$, and ${ }^{58} \mathrm{Mn}$. (A color version of this figure is available in the online journal.)

et al. (2000), we can see the density values at which these nuclei strongly affect the stellar evolution of these points, representing the nuclei with higher contribution to $\dot{Y}_{e}$, as it was indicated by Auf. We stress that we used the same 
Table 3

Electron Capture for the 16 More Relevant Nuclei at $Y_{e}=0.45$

\begin{tabular}{|c|c|c|c|c|c|c|}
\hline Nucleus & $\begin{array}{c}\lambda_{\mathrm{EC}}(\mathrm{GT}) \\
\left(\mathrm{s}^{-1}\right)\end{array}$ & $\begin{array}{c}\lambda_{\mathrm{EC}}(\mathrm{SM}) \\
\left(\mathrm{s}^{-1}\right)\end{array}$ & $\begin{array}{c}\lambda_{\mathrm{EC}}(\text { Auf }) \\
\left(\mathrm{s}^{-1}\right)\end{array}$ & $\begin{array}{c}\lambda_{\mathrm{EC}}(\mathrm{FFN}) \\
\left(\mathrm{s}^{-1}\right)\end{array}$ & $\begin{array}{c}\left|\dot{Y}_{e \mathrm{EC}}(\mathrm{GT})\right| \\
\left(\mathrm{s}^{-1}\right)\end{array}$ & $\begin{array}{c}\mid \dot{Y}_{e \mathrm{EC}}(\text { Auf }) \mid \\
\left(\mathrm{s}^{-1}\right)\end{array}$ \\
\hline${ }^{58} \mathrm{Co}$ & $4.00 \times 10^{-4}$ & $3.30 \times 10^{-4}$ & $3.07 \times 10^{-2}$ & $1.55 \times 10^{-2}$ & $1.83 \times 10^{-9}$ & $1.48 \times 10^{-8}$ \\
\hline${ }^{55} \mathrm{Fe}$ & $3.54 \times 10^{-4}$ & $\ldots$ & $6.00 \times 10^{-3}$ & $1.21 \times 10^{-8}$ & $1.23 \times 10^{-9}$ & $1.25 \times 10^{-8}$ \\
\hline${ }^{60} \mathrm{Co}$ & $2.76 \times 10^{-5}$ & $1.00 \times 10^{-4}$ & $1.27 \times 10^{-2}$ & $1.15 \times 10^{-2}$ & $9.41 \times 10^{-10}$ & $1.27 \times 10^{-7}$ \\
\hline${ }^{50} \mathrm{~V}$ & $1.36 \times 10^{-3}$ & $\ldots$ & $2.45 \times 10^{-2}$ & $3.05 \times 10^{-3}$ & $8.31 \times 10^{-10}$ & $2.60 \times 10^{-9}$ \\
\hline${ }^{57} \mathrm{Co}$ & $5.40 \times 10^{-4}$ & $\ldots$ & $1.29 \times 10^{-2}$ & $1.04 \times 10^{-2}$ & $4.97 \times 10^{-10}$ & $8.67 \times 10^{-9}$ \\
\hline${ }^{51} \mathrm{Cr}$ & $8.47 \times 10^{-4}$ & $\ldots$ & $9.33 \times 10^{-3}$ & $5.20 \times 10^{-3}$ & $2.94 \times 10^{-10}$ & $1.36 \times 10^{-9}$ \\
\hline${ }^{59} \mathrm{Ni}$ & $1.17 \times 10^{-3}$ & $\ldots$ & $\ldots$ & $\ldots$ & $1.46 \times 10^{-10}$ & $\ldots$ \\
\hline${ }^{63} \mathrm{Cu}$ & $9.89 \times 10^{-11}$ & $\ldots$ & $1.85 \times 10^{-2}$ & $\cdots$ & $9.59 \times 10^{-11}$ & $1.53 \times 10^{-8}$ \\
\hline${ }^{56} \mathrm{Co}$ & $6.08 \times 10^{-3}$ & $3.00 \times 10^{-3}$ & $\ldots$ & $\ldots$ & $4.70 \times 10^{-11}$ & $\ldots$ \\
\hline${ }^{61} \mathrm{Ni}$ & $3.72 \times 10^{-7}$ & $\ldots$ & $1.20 \times 10^{-3}$ & $\ldots$ & $2.02 \times 10^{-11}$ & $2.86 \times 10^{-8}$ \\
\hline${ }^{62} \mathrm{Cu}$ & $1.07 \times 10^{-3}$ & $\cdots$ & $4.60 \times 10^{+0}$ & $\ldots$ & $1.07 \times 10^{-11}$ & $3.77 \times 10^{-9}$ \\
\hline${ }^{59} \mathrm{Co}$ & $4.35 \times 10^{-8}$ & $\cdots$ & $6.57 \times 10^{-4}$ & $5.44 \times 10^{-4}$ & $7.60 \times 10^{-12}$ & $1.27 \times 10^{-7}$ \\
\hline${ }^{61} \mathrm{Cu}$ & $2.02 \times 10^{-3}$ & $\cdots$ & $\ldots$ & $\ldots$ & $1.64 \times 10^{-12}$ & .. \\
\hline${ }^{58} \mathrm{Ni}$ & $9.85 \times 10^{-5}$ & $\ldots$ & $\ldots$ & $\ldots$ & $5.08 \times 10^{-13}$ & $\ldots$ \\
\hline${ }^{55} \mathrm{Co}$ & $5.81 \times 10^{-3}$ & $\ldots$ & $\ldots$ & $\ldots$ & $3.00 \times 10^{-13}$ & $\ldots$ \\
\hline \multirow[t]{2}{*}{${ }^{56} \mathrm{Mn}$} & $3.36 \times 10^{-9}$ & $9.90 \times 10^{-5}$ & $2.56 \times 10^{-4}$ & $4.29 \times 10^{-5}$ & $2.88 \times 10^{-13}$ & $3.22 \times 10^{-9}$ \\
\hline & & $\rho_{7}=14.5$ & $T_{9}=3.8$ & & $\begin{array}{c}\dot{Y}_{e \mathrm{EC}}(\mathrm{GT}) \\
-5.91 \times 10^{-9}\end{array}$ & $\begin{array}{c}\dot{Y}_{e \mathrm{EC}}(\text { Auf }) \\
-4.96 \times 10^{-7}\end{array}$ \\
\hline
\end{tabular}

Table 4

Electron Capture for the 11 More Relevant Nuclei at $Y_{e}=0.44$

\begin{tabular}{|c|c|c|c|c|c|c|}
\hline Nucleus & $\begin{array}{c}\lambda \lambda_{\mathrm{EC}}(\mathrm{GT}) \\
\left(\mathrm{s}^{-1}\right)\end{array}$ & $\begin{array}{c}\lambda_{\mathrm{EC}}(\mathrm{SM}) \\
\left(\mathrm{s}^{-1}\right)\end{array}$ & $\begin{array}{c}\lambda_{\mathrm{EC}} \text { (Auf) } \\
\left(\mathrm{s}^{-1}\right)\end{array}$ & $\begin{array}{c}\lambda_{\mathrm{EC}}(\mathrm{FFN}) \\
\left(\mathrm{s}^{-1}\right)\end{array}$ & $\begin{array}{c}\left|\dot{Y}_{e \mathrm{EC}}(\mathrm{GT})\right| \\
\left(\mathrm{s}^{-1}\right)\end{array}$ & $\begin{array}{c}\mid \dot{Y}_{e \mathrm{EC}}(\text { Auf }) \mid \\
\left(\mathrm{s}^{-1}\right)\end{array}$ \\
\hline${ }^{60} \mathrm{Co}$ & $2.02 \times 10^{-4}$ & $1.80 \times 10^{-4}$ & $6.10 \times 10^{-2}$ & $1.10 \times 10^{-1}$ & $3.92 \times 10^{-9}$ & $3.31 \times 10^{-7}$ \\
\hline${ }^{50} \mathrm{~V}$ & $4.87 \times 10^{-3}$ & $\ldots$ & $\ldots$ & $\ldots$ & $3.71 \times 10^{-10}$ & $\ldots$ \\
\hline${ }^{56} \mathrm{Mn}$ & $1.26 \times 10^{-6}$ & $2.30 \times 10^{-4}$ & $2.00 \times 10^{-3}$ & $4.10 \times 10^{-3}$ & $1.62 \times 10^{-10}$ & $3.92 \times 10^{-8}$ \\
\hline${ }^{59} \mathrm{Co}$ & $9.24 \times 10^{-6}$ & $\ldots$ & $5.07 \times 10^{-3}$ & $\ldots$ & $9.06 \times 10^{-11}$ & $4.43 \times 10^{-8}$ \\
\hline${ }^{58} \mathrm{Co}$ & $1.43 \times 10^{-3}$ & $\ldots$ & $9.07 \times 10^{-2}$ & $\ldots$ & $8.44 \times 10^{-11}$ & $4.39 \times 10^{-10}$ \\
\hline${ }^{61} \mathrm{Ni}$ & $1.77 \times 10^{-5}$ & $\ldots$ & $8.38 \times 10^{-3}$ & $\ldots$ & $3.73 \times 10^{-11}$ & $6.03 \times 10^{-9}$ \\
\hline${ }^{63} \mathrm{Cu}$ & $6.69 \times 10^{-4}$ & $\ldots$ & $8.42 \times 10^{-2}$ & $\ldots$ & $1.99 \times 10^{-11}$ & $1.69 \times 10^{-9}$ \\
\hline${ }^{55} \mathrm{Fe}$ & $1.95 \times 10^{-3}$ & $\ldots$ & $3.13 \times 10^{-2}$ & $\ldots$ & $1.73 \times 10^{-11}$ & $7.48 \times 10^{-11}$ \\
\hline${ }^{51} \mathrm{Cr}$ & $4.06 \times 10^{-3}$ & $\ldots$ & $\ldots$ & $\ldots$ & $1.30 \times 10^{-11}$ & $\ldots$ \\
\hline${ }^{52} \mathrm{~V}$ & $1.17 \times 10^{-6}$ & $\ldots$ & $9.40 \times 10^{-4}$ & $\ldots$ & $1.20 \times 10^{-11}$ & $1.22 \times 10^{-8}$ \\
\hline \multirow[t]{2}{*}{${ }^{57} \mathrm{Co}$} & $2.60 \times 10^{-3}$ & $\cdots$ & $\cdots$ & $\cdots$ & $4.90 \times 10^{-12}$ & $\cdots$ \\
\hline & & $\rho_{7}=33$ & $T_{9}=4.24$ & & $\begin{array}{c}\dot{Y}_{e \mathrm{EC}}(\mathrm{GT}) \\
-4.73 \times 10^{-9}\end{array}$ & $\begin{array}{c}\dot{Y}_{e \mathrm{EC}}(\text { Auf }) \\
-5.73 \times 10^{-7}\end{array}$ \\
\hline
\end{tabular}

Table 5

Electron Capture for the 12 More Relevant Nuclei at $Y_{e}=0.43$

\begin{tabular}{|c|c|c|c|c|c|c|}
\hline Nucleus & $\begin{array}{c}\lambda_{\mathrm{EC}}(\mathrm{GT}) \\
\left(\mathrm{s}^{-1}\right)\end{array}$ & $\begin{array}{c}\lambda_{\mathrm{EC}}(\mathrm{SM}) \\
\left(\mathrm{s}^{-1}\right)\end{array}$ & $\begin{array}{c}\lambda_{\mathrm{EC}}(\text { Auf }) \\
\left(\mathrm{s}^{-1}\right)\end{array}$ & $\begin{array}{c}\lambda_{\mathrm{EC}}(\mathrm{FFN}) \\
\left(\mathrm{s}^{-1}\right)\end{array}$ & $\begin{array}{c}\left|\dot{Y}_{e \mathrm{EC}}(\mathrm{GT})\right| \\
\left(\mathrm{s}^{-1}\right)\end{array}$ & $\begin{array}{c}\mid \dot{Y}_{e \mathrm{EC}}(\text { Auf }) \mid \\
\left(\mathrm{s}^{-1}\right)\end{array}$ \\
\hline$\overline{{ }^{60} \mathrm{Co}}$ & $4.27 \times 10^{-3}$ & $8.00 \times 10^{-4}$ & $2.31 \times 10^{+0}$ & $2.31 \times 10^{+0}$ & $1.80 \times 10^{-8}$ & $3.66 \times 10^{-6}$ \\
\hline${ }^{56} \mathrm{Mn}$ & $2.61 \times 10^{-4}$ & $4.80 \times 10^{-4}$ & $2.90 \times 10^{-2}$ & $9.29 \times 10^{-3}$ & $1.28 \times 10^{-8}$ & $2.91 \times 10^{-7}$ \\
\hline${ }^{52} \mathrm{~V}$ & $1.17 \times 10^{-5}$ & $\ldots$ & $1.81 \times 10^{-2}$ & $6.11 \times 10^{-3}$ & $1.16 \times 10^{-8}$ & $2.58 \times 10^{-7}$ \\
\hline${ }^{51} \mathrm{~V}$ & $1.67 \times 10^{-4}$ & $\ldots$ & $9.71 \times 10^{-3}$ & $4.40 \times 10^{-3}$ & $2.32 \times 10^{-9}$ & $1.53 \times 10^{-7}$ \\
\hline${ }^{62} \mathrm{Co}$ & $2.75 \times 10^{-5}$ & $\ldots$ & $3.22 \times 10^{-2}$ & & $1.69 \times 10^{-9}$ & $5.34 \times 10^{-7}$ \\
\hline${ }^{59} \mathrm{Co}$ & $1.67 \times 10^{-3}$ & $\ldots$ & $3.35 \times 10^{-1}$ & $4.35 \times 10^{-1}$ & $8.86 \times 10^{-10}$ & $2.51 \times 10^{-7}$ \\
\hline${ }^{50} \mathrm{~V}$ & $4.10 \times 10^{-2}$ & $\ldots$ & $\ldots$ & $\ldots$ & $6.87 \times 10^{-10}$ & $\ldots$ \\
\hline${ }^{55} \mathrm{Mn}$ & $4.26 \times 10^{-5}$ & $\ldots$ & $8.73 \times 10^{-3}$ & $9.10 \times 10^{-3}$ & $5.53 \times 10^{-10}$ & $7.70 \times 10^{-8}$ \\
\hline${ }^{61} \mathrm{Ni}$ & $1.49 \times 10^{-3}$ & $\ldots$ & $5.07 \times 10^{-1}$ & $\ldots$ & $1.50 \times 10^{-10}$ & $2.83 \times 10^{-8}$ \\
\hline${ }^{57} \mathrm{Fe}$ & $1.01 \times 10^{-5}$ & $\ldots$ & $\ldots$ & $\ldots$ & $5.86 \times 10^{-11}$ & $\ldots$ \\
\hline${ }^{58} \mathrm{Co}$ & $1.21 \times 10^{-2}$ & $9.00 \times 10^{-4}$ & $\ldots$ & $\ldots$ & $3.14 \times 10^{-11}$ & $\ldots$ \\
\hline \multirow[t]{2}{*}{${ }^{63} \mathrm{Cu}$} & $1.32 \times 10^{-2}$ & $\cdots$ & $\cdots$ & $\cdots$ & $1.97 \times 10^{-11}$ & $\cdots$ \\
\hline & & $\rho_{7}=106$ & $T_{9}=4.93$ & & $\begin{array}{c}\dot{Y}_{e \mathrm{EC}}(\mathrm{GT}) \\
-4.89 \times 10^{-8}\end{array}$ & $\begin{array}{c}\dot{Y}_{e \mathrm{EC}}(\text { Auf }) \\
-8.21 \times 10^{-6}\end{array}$ \\
\hline
\end{tabular}

density, temperature, and chemical potential parameterization as Aufderheide et al. (1994b) and Martínez-Pinedo et al. (2000), and the only difference was the nuclear model used to evaluate the decay rates. Langanke and Martínez-Pinedo have pointed out in Langanke \& Martínez-Pinedo (1999) that the EC rates of six nuclei were overestimated by Auf (Aufderheide et al. 1994b) and FFN (Fuller et al. 1982a). The ${ }^{54} \mathrm{Mn}$ nucleus is the only one of those six nuclei not included in our sample. For the other five, 
Table 6

Electron Capture for the 13 More Relevant Nuclei at $Y_{e}=0.425$

\begin{tabular}{|c|c|c|c|c|c|c|}
\hline Nucleus & $\begin{array}{c}\lambda_{\mathrm{EC}}(\mathrm{GT}) \\
\left(\mathrm{s}^{-1}\right)\end{array}$ & $\begin{array}{c}\lambda_{\mathrm{EC}}(\mathrm{SM}) \\
\left(\mathrm{s}^{-1}\right)\end{array}$ & $\begin{array}{c}\lambda_{\mathrm{EC}}(\text { Auf }) \\
\left(\mathrm{s}^{-1}\right)\end{array}$ & $\begin{array}{c}\lambda_{\mathrm{EC}}(\mathrm{FFN}) \\
\left(\mathrm{s}^{-1}\right)\end{array}$ & $\begin{array}{c}\left|\dot{Y}_{e \mathrm{EC}}(\mathrm{GT})\right| \\
\left(\mathrm{s}^{-1}\right)\end{array}$ & $\begin{array}{c}\mid \dot{Y}_{e \mathrm{EC}}(\text { Auf }) \mid \\
\left(\mathrm{s}^{-1}\right)\end{array}$ \\
\hline $52 \mathrm{~V}$ & $2.20 \times 10^{-3}$ & $\ldots$ & $9.62 \times 10^{-2}$ & $\cdots$ & $1.11 \times 10^{-7}$ & $1.04 \times 10^{-6}$ \\
\hline${ }^{56} \mathrm{Mn}$ & $6.74 \times 10^{-7}$ & $1.00 \times 10^{-2}$ & $1.38 \times 10^{-1}$ & $1.00 \times 10^{-1}$ & $6.26 \times 10^{-8}$ & $7.58 \times 10^{-7}$ \\
\hline${ }^{62} \mathrm{Co}$ & $5.65 \times 10^{-5}$ & $\ldots$ & $2.51 \times 10^{-1}$ & $\ldots$ & $4.16 \times 10^{-8}$ & $3.86 \times 10^{-6}$ \\
\hline${ }^{60} \mathrm{Co}$ & $7.95 \times 10^{-8}$ & $\ldots$ & $6.83 \times 10^{+0}$ & $\cdots$ & $3.55 \times 10^{-8}$ & $4.84 \times 10^{-6}$ \\
\hline${ }^{51} \mathrm{~V}$ & $3.52 \times 10^{-3}$ & $\ldots$ & $6.58 \times 10^{-2}$ & $\ldots$ & $2.49 \times 10^{-8}$ & $4.81 \times 10^{-7}$ \\
\hline${ }^{55} \mathrm{Mn}$ & $1.60 \times 10^{-3}$ & $\ldots$ & $\ldots$ & $\ldots$ & $5.16 \times 10^{-9}$ & $\ldots$ \\
\hline${ }^{59} \mathrm{Co}$ & $1.85 \times 10^{-2}$ & $\ldots$ & $1.85 \times 10^{+0}$ & $\ldots$ & $1.96 \times 10^{-9}$ & $3.94 \times 10^{-7}$ \\
\hline${ }^{53} \mathrm{Cr}$ & $2.34 \times 10^{-4}$ & $\ldots$ & $\ldots$ & $\ldots$ & $1.09 \times 10^{-9}$ & $\ldots$ \\
\hline${ }^{50} \mathrm{~V}$ & $1.54 \times 10^{-1}$ & $\ldots$ & $\ldots$ & $\ldots$ & $1.07 \times 10^{-9}$ & $\ldots$ \\
\hline${ }^{61} \mathrm{Co}$ & $3.04 \times 10^{-5}$ & $\ldots$ & $2.74 \times 10^{-2}$ & $\ldots$ & $9.65 \times 10^{-10}$ & $1.41 \times 10^{-6}$ \\
\hline${ }^{57} \mathrm{Fe}$ & $4.22 \times 10^{-4}$ & $\ldots$ & $\ldots$ & $\ldots$ & $5.41 \times 10^{-10}$ & $\ldots$ \\
\hline${ }^{63} \mathrm{Ni}$ & $2.64 \times 10^{-5}$ & $\ldots$ & $4.36 \times 10^{-2}$ & $\ldots$ & $5.10 \times 10^{-10}$ & $3.91 \times 10^{-7}$ \\
\hline \multirow[t]{2}{*}{${ }^{58} \mathrm{Mn}$} & $3.23 \times 10^{-6}$ & $\ldots$ & $3.42 \times 10^{-2}$ & $\ldots$ & $2.55 \times 10^{-10}$ & $1.11 \times 10^{-6}$ \\
\hline & & $\rho_{7}=220$ & $T_{9}=5.39$ & & $\begin{array}{c}\dot{Y}_{e \mathrm{EC}}(\mathrm{GT}) \\
-2.88 \times 10^{-7}\end{array}$ & $\begin{array}{c}\dot{Y}_{e \mathrm{EC}}(\text { Auf }) \\
-2.84 \times 10^{-5}\end{array}$ \\
\hline
\end{tabular}

Table 7

Electron Capture for the 18 More Relevant Nuclei at $Y_{e}=0.41$

\begin{tabular}{lcccccc}
\hline \hline Nucleus & $\begin{array}{c}\lambda_{\mathrm{EC}}(\mathrm{GT}) \\
\left(\mathrm{s}^{-1}\right)\end{array}$ & $\begin{array}{c}\lambda_{\mathrm{EC}}(\mathrm{SM}) \\
\left(\mathrm{s}^{-1}\right)\end{array}$ & $\begin{array}{c}\lambda_{\mathrm{EC}}(\text { Auf }) \\
\left(\mathrm{s}^{-1}\right)\end{array}$ & $\begin{array}{c}\lambda_{\mathrm{EC}}(\mathrm{FFN}) \\
\left(\mathrm{s}^{-1}\right)\end{array}$ & $\begin{array}{c}\left|\dot{Y}_{e \mathrm{EC}}(\mathrm{GT})\right| \\
\left(\mathrm{s}^{-1}\right)\end{array}$ & $\begin{array}{c}\left|\dot{Y}_{e \mathrm{EC}}(\mathrm{Auf})\right| \\
\left(\mathrm{s}^{-1}\right)\end{array}$ \\
\hline${ }^{50} \mathrm{Sc}$ & $3.20 \times 10^{+0}$ & $\ldots$ & $2.55 \times 10^{+1}$ & $\ldots$ & $2.37 \times 10^{-3}$ & $5.26 \times 10^{-3}$ \\
${ }^{51} \mathrm{Sc}$ & $3.16 \times 10^{-1}$ & $\ldots$ & $\ldots$ & $\ldots$ & $3.65 \times 10^{-4}$ & $\ldots$ \\
${ }^{54} \mathrm{~V}$ & $3.48 \times 10^{+0}$ & $\ldots$ & $9.66 \times 10^{+1}$ & $\ldots$ & $2.17 \times 10^{-4}$ & $3.85 \times 10^{-3}$ \\
${ }^{53} \mathrm{~V}$ & $2.05 \times 10^{+0}$ & $\ldots$ & $9.23 \times 10^{+1}$ & $\ldots$ & $1.60 \times 10^{-4}$ & $9.16 \times 10^{-3}$ \\
${ }^{51} \mathrm{Ti}$ & $9.48 \times 10^{-1}$ & $\ldots$ & $\ldots$ & $\ldots$ & $1.56 \times 10^{-4}$ & $\ldots$ \\
${ }^{63} \mathrm{Co}$ & $1.20 \times 10^{+0}$ & $\ldots$ & $1.62 \times 10^{-2}$ & $\ldots$ & $8.10 \times 10^{-5}$ & $1.23 \times 10^{-2}$ \\
${ }^{65} \mathrm{Ni}$ & $5.35 \times 10^{-1}$ & $\ldots$ & $1.44 \times 10^{+2}$ & $\ldots$ & $4.73 \times 10^{-5}$ & $9.82 \times 10^{-3}$ \\
${ }^{58} \mathrm{Mn}$ & $1.24 \times 10^{+0}$ & $7.00 \times 10^{-3}$ & $1.05 \times 10^{+3}$ & $7.90 \times 10^{+2}$ & $2.94 \times 10^{-5}$ & $1.79 \times 10^{-2}$ \\
${ }^{59} \mathrm{Mn}$ & $2.61 \times 10^{-1}$ & $\ldots$ & $1.41 \times 10^{+2}$ & $\ldots$ & $2.79 \times 10^{-5}$ & $7.53 \times 10^{-3}$ \\
${ }^{55} \mathrm{Cr}$ & $6.65 \times 10^{-1}$ & $\ldots$ & $\ldots$ & $\ldots$ & $2.43 \times 10^{-5}$ & $\ldots$ \\
${ }^{61} \mathrm{Fe}$ & $1.42 \times 10^{-1}$ & $\ldots$ & $1.63 \times 10^{+2}$ & $\ldots$ & $2.17 \times 10^{-5}$ & $1.63 \times 10^{-2}$ \\
${ }^{57} \mathrm{Mn}$ & $1.36 \times 10^{+0}$ & $\ldots$ & $8.36 \times 10^{+2}$ & $4.29 \times 10^{+2}$ & $1.85 \times 10^{-5}$ & $1.01 \times 10^{-2}$ \\
${ }^{66} \mathrm{Ni}$ & $1.35 \times 10^{-2}$ & $\ldots$ & $\ldots$ & $\ldots$ & $1.63 \times 10^{-5}$ & $\ldots$ \\
${ }^{52} \mathrm{~V}$ & $3.98 \times 10^{+0}$ & $\ldots$ & $\ldots$ & $\ldots$ & $1.58 \times 10^{-5}$ & $\ldots$ \\
${ }^{62} \mathrm{Co}$ & $2.78 \times 10^{+0}$ & $\ldots$ & $1.44 \times 10^{+3}$ & $\ldots$ & $1.48 \times 10^{-5}$ & $5.68 \times 10^{-3}$ \\
${ }^{60} \mathrm{Mn}$ & $2.18 \times 10^{-1}$ & $\ldots$ & $2.55 \times 10^{+2}$ & $\ldots$ & $5.66 \times 10^{-6}$ & $6.32 \times 10^{-3}$ \\
${ }^{59} \mathrm{Fe}$ & $6.44 \times 10^{-1}$ & $\ldots$ & $7.20 \times 10^{+2}$ & $7.43 \times 10^{+2}$ & $5.34 \times 10^{-6}$ & $6.17 \times 10^{-3}$ \\
${ }^{61} \mathrm{Co}$ & $6.67 \times 10^{+0}$ & $\ldots$ & $1.15 \times 10^{+3}$ & $\ldots$ & $4.92 \times 10^{-6}$ & $3.93 \times 10^{-3}$ \\
\hline & & $\ldots$ & & & $\dot{Y}_{e \mathrm{EC}}(\mathrm{GT})$ & $\dot{Y}_{e \mathrm{EC}}(\mathrm{Auf})$ \\
& & $\ldots$ & & & $-4.89 \times 10^{-8}$ & $-2.23 \times 10^{-1}$ \\
\hline
\end{tabular}

Table 8

Beta Decay for the Five More Relevant Nuclei at $Y_{e}=0.485$

\begin{tabular}{|c|c|c|c|c|c|c|}
\hline Nucleus & $\begin{array}{c}\lambda_{\mathrm{DB}}(\mathrm{GT}) \\
\left(\mathrm{s}^{-1}\right)\end{array}$ & $\begin{array}{c}\lambda_{\mathrm{DB}}(\mathrm{SM}) \\
\left(\mathrm{s}^{-1}\right)\end{array}$ & $\begin{array}{c}\lambda_{\mathrm{DB}}(\mathrm{Auf}) \\
\left(\mathrm{s}^{-1}\right)\end{array}$ & $\begin{array}{c}\lambda_{\mathrm{DB}}(\mathrm{FFN}) \\
\left(\mathrm{s}^{-1}\right)\end{array}$ & $\begin{array}{c}\left|\dot{Y}_{e \mathrm{DB}}(\mathrm{GT})\right| \\
\left(\mathrm{s}^{-1}\right)\end{array}$ & $\mid \begin{array}{c}\left|\dot{Y}_{e \mathrm{DB}}(\mathrm{Auf})\right| \\
\left(\mathrm{s}^{-1}\right)\end{array}$ \\
\hline$\overline{56} \mathrm{Mn}$ & $2.79 \times 10^{-4}$ & $2.50 \times 10^{-3}$ & $1.16 \times 10^{-2}$ & $1.00 \times 10^{-2}$ & $8.60 \times 10^{-17}$ & $1.48 \times 10^{-18}$ \\
\hline${ }^{60} \mathrm{Co}$ & $6.15 \times 10^{-5}$ & $\ldots$ & $4.03 \times 10^{-3}$ & $\ldots$ & $7.39 \times 10^{-17}$ & $5.06 \times 10^{-18}$ \\
\hline${ }^{52} \mathrm{~V}$ & $4.89 \times 10^{-4}$ & $\ldots$ & $\ldots$ & $\ldots$ & $3.20 \times 10^{-18}$ & $\ldots$ \\
\hline${ }^{50} \mathrm{~V}$ & $4.49 \times 10^{-9}$ & $\ldots$ & $\ldots$ & $\ldots$ & $2.62 \times 10^{-18}$ & $\ldots$ \\
\hline \multirow[t]{2}{*}{${ }^{57} \mathrm{Mn}$} & $4.88 \times 10^{-5}$ & $\ldots$ & $\cdots$ & $\ldots$ & $3.67 \times 10^{-21}$ & $\cdots$ \\
\hline & & $\rho_{7}=4.32$ & $T_{9}=3.26$ & & $\begin{array}{c}\dot{Y}_{e \mathrm{DB}}(\mathrm{GT}) \\
1.66 \times 10^{-16}\end{array}$ & $\begin{array}{c}\dot{Y}_{e \mathrm{DB}}(\text { Auf }) \\
2.08 \times 10^{-13}\end{array}$ \\
\hline
\end{tabular}

we observe that ${ }^{56} \mathrm{Co},{ }^{58} \mathrm{Co}$, and ${ }^{60} \mathrm{Co}$ show similar values to the $\mathrm{SM}$ ones. However, ${ }^{58} \mathrm{Mn}$ presents a value that is three orders of magnitude below the FFN and Auf results, but two orders above SM ones, and ${ }^{56} \mathrm{Mn}$ shows six orders of magnitude below FFN and Auf, but two below SM results. For these last two nuclei, we do not have overestimated rates, in spite of the values that do not agree with the SM ones, as it happens with cobalt, which we also compared. The compared values are for those points of the stellar trajectory in which the nuclei have a major influence through their rate. We have observed that for other $Y_{e}$ values, the GT calculates rates that are sometimes smaller and in other cases bigger when compared with SM results. Figure 7 shows the above discussion for ${ }^{56} \mathrm{Co}$ and ${ }^{58} \mathrm{Co}$. We emphasize that this behavior is irrelevant when we consider that the GT 
Table 9

Beta Decay for the Eight More Relevant Nuclei at $Y_{e}=0.47$

\begin{tabular}{lcccccc}
\hline \hline Nucleus & $\begin{array}{c}\lambda_{\mathrm{DB}}(\mathrm{GT}) \\
\left(\mathrm{s}^{-1}\right)\end{array}$ & $\begin{array}{c}\lambda_{\mathrm{DB}}(\mathrm{SM}) \\
\left(\mathrm{s}^{-1}\right)\end{array}$ & $\begin{array}{c}\lambda_{\mathrm{DB}}(\mathrm{Auf}) \\
\left(\mathrm{s}^{-1}\right)\end{array}$ & $\begin{array}{c}\lambda_{\mathrm{DB}}(\mathrm{FFN}) \\
\left(\mathrm{s}^{-1}\right)\end{array}$ & $\begin{array}{c}\left|\dot{Y}_{e \mathrm{DB}}(\mathrm{GT})\right| \\
\left(\mathrm{s}^{-1}\right)\end{array}$ & $\begin{array}{c}\left|\dot{Y}_{e \mathrm{DB}}(\mathrm{Auf})\right| \\
\left(\mathrm{s}^{-1}\right)\end{array}$ \\
\hline${ }^{56} \mathrm{Mn}$ & $2.37 \times 10^{-4}$ & $3.00 \times 10^{-3}$ & $1.09 \times 10^{-2}$ & $3.82 \times 10^{-3}$ & $4.23 \times 10^{-13}$ & $3.87 \times 10^{-12}$ \\
${ }^{60} \mathrm{Co}$ & $4.93 \times 10^{-5}$ & $\ldots$ & $4.66 \times 10^{-3}$ & $1.09 \times 10^{-3}$ & $2.17 \times 10^{-13}$ & $7.94 \times 10^{-12}$ \\
${ }^{52} \mathrm{~V}$ & $4.32 \times 10^{-4}$ & $\ldots$ & $1.60 \times 10^{-2}$ & $2.07 \times 10^{-3}$ & $2.76 \times 10^{-14}$ & $1.33 \times 10^{-13}$ \\
${ }^{50} \mathrm{~V}$ & $3.20 \times 10^{-9}$ & $\ldots$ & $1.89 \times 10^{-5}$ & $4.03 \times 10^{-6}$ & $2.06 \times 10^{-16}$ & $1.79 \times 10^{-13}$ \\
${ }^{57} \mathrm{Mn}$ & $2.97 \times 10^{-5}$ & $\ldots$ & $\ldots$ & $\ldots$ & $1.72 \times 10^{-16}$ & $\ldots$ \\
${ }^{55} \mathrm{Cr}$ & $1.03 \times 10^{-4}$ & $\ldots$ & $\ldots$ & $\ldots$ & $5.92 \times 10^{-17}$ & $\ldots$ \\
${ }^{53} \mathrm{~V}$ & $4.84 \times 10^{-4}$ & $\ldots$ & $\ldots$ & $\ldots$ & $3.73 \times 10^{-17}$ & $\ldots$ \\
${ }^{59} \mathrm{Fe}$ & $7.29 \times 10^{-7}$ & $3.50 \times 10^{-5}$ & $6.95 \times 10^{-3}$ & $5.11 \times 10^{-3}$ & $1.15 \times 10^{-17}$ & $2.43 \times 10^{-13}$ \\
\hline & $\rho_{7}=5.86$ & $T_{9}=3.4$ & & & $\dot{Y}_{\text {DB }}(\mathrm{GT})$ & $\dot{Y}_{e \mathrm{DB}}(\mathrm{Auf})$ \\
& & & & & $6.68 \times 10^{-13}$ & $1.24 \times 10^{-10}$ \\
\hline
\end{tabular}

Table 10

Beta Decay for the 15 More Relevant Nuclei at $Y_{e}=0.45$

\begin{tabular}{|c|c|c|c|c|c|c|}
\hline Nucleus & $\begin{array}{c}\lambda_{\mathrm{DB}}(\mathrm{GT}) \\
\left(\mathrm{s}^{-1}\right)\end{array}$ & $\begin{array}{c}\lambda_{\mathrm{DB}}(\mathrm{SM}) \\
\left(\mathrm{s}^{-1}\right)\end{array}$ & $\begin{array}{c}\lambda_{\mathrm{DB}}(\mathrm{Auf}) \\
\left(\mathrm{s}^{-1}\right)\end{array}$ & $\begin{array}{c}\lambda_{\mathrm{DB}}(\mathrm{FFN}) \\
\left(\mathrm{s}^{-1}\right)\end{array}$ & $\begin{array}{c}\left|\dot{Y}_{e \mathrm{DB}}(\mathrm{GT})\right| \\
\left(\mathrm{s}^{-1}\right)\end{array}$ & $\begin{array}{c}\mid \dot{Y}_{e \mathrm{DB}}(\text { Auf }) \mid \\
\left(\mathrm{s}^{-1}\right)\end{array}$ \\
\hline$\overline{5^{3} \mathrm{~V}}$ & $2.38 \times 10^{-4}$ & $\cdots$ & $5.60 \times 10^{-3}$ & $5.58 \times 10^{-3}$ & $1.60 \times 10^{-8}$ & $9.99 \times 10^{-9}$ \\
\hline${ }^{56} \mathrm{Mn}$ & $1.19 \times 10^{-4}$ & $6.50 \times 10^{-3}$ & $7.99 \times 10^{-3}$ & $2.52 \times 10^{-3}$ & $1.10 \times 10^{-8}$ & $1.00 \times 10^{-7}$ \\
\hline${ }^{52} \mathrm{~V}$ & $2.46 \times 10^{-4}$ & $\ldots$ & $1.23 \times 10^{-2}$ & $1.36 \times 10^{-3}$ & $5.51 \times 10^{-9}$ & $2.77 \times 10^{-8}$ \\
\hline${ }^{62} \mathrm{Cr}$ & $2.95 \times 10^{-3}$ & $\ldots$ & $7.87 \times 10^{-2}$ & $\ldots$ & $2.28 \times 10^{-9}$ & $9.41 \times 10^{-9}$ \\
\hline${ }^{55} \mathrm{Cr}$ & $3.80 \times 10^{-5}$ & $\ldots$ & $1.52 \times 10^{-3}$ & $4.21 \times 10^{-3}$ & $1.09 \times 10^{-9}$ & $1.61 \times 10^{-8}$ \\
\hline${ }^{58} \mathrm{Mn}$ & $9.16 \times 10^{-3}$ & $\ldots$ & $1.71 \times 10^{-1}$ & $1.30 \times 10^{-1}$ & $1.07 \times 10^{-9}$ & $3.54 \times 10^{-9}$ \\
\hline${ }^{57} \mathrm{Mn}$ & $1.59 \times 10^{-5}$ & $\ldots$ & $1.36 \times 10^{-3}$ & $1.91 \times 10^{-2}$ & $7.24 \times 10^{-10}$ & $1.60 \times 10^{-8}$ \\
\hline${ }^{60} \mathrm{Co}$ & $1.92 \times 10^{-5}$ & $6.00 \times 10^{-4}$ & $4.54 \times 10^{-3}$ & $1.58 \times 10^{-3}$ & $6.56 \times 10^{-10}$ & $4.56 \times 10^{-8}$ \\
\hline${ }^{54} \mathrm{~V}$ & $5.56 \times 10^{-2}$ & $\ldots$ & $\ldots$ & $\ldots$ & $3.55 \times 10^{-10}$ & $\ldots$ \\
\hline${ }^{51} \mathrm{Ti}$ & $4.11 \times 10^{-5}$ & $\ldots$ & $1.07 \times 10^{-3}$ & $1.55 \times 10^{-3}$ & $2.20 \times 10^{-10}$ & $2.70 \times 10^{-9}$ \\
\hline${ }^{50} \mathrm{Sc}$ & $5.91 \times 10^{-2}$ & $\ldots$ & $\ldots$ & $\ldots$ & $2.05 \times 10^{-10}$ & $\ldots$ \\
\hline${ }^{63} \mathrm{Co}$ & $8.70 \times 10^{-4}$ & $\ldots$ & $2.05 \times 10^{-2}$ & $\ldots$ & $1.75 \times 10^{-10}$ & $2.26 \times 10^{-9}$ \\
\hline${ }^{61} \mathrm{Fe}$ & $1.64 \times 10^{-3}$ & $\ldots$ & $8.64 \times 10^{-2}$ & $\ldots$ & $1.45 \times 10^{-10}$ & $1.85 \times 10^{-9}$ \\
\hline${ }^{59} \mathrm{Mn}$ & $9.19 \times 10^{-3}$ & $8.00 \times 10^{-3}$ & $\ldots$ & $\ldots$ & $4.94 \times 10^{-11}$ & $\ldots$ \\
\hline \multirow[t]{2}{*}{${ }^{59} \mathrm{Fe}$} & $1.96 \times 10^{-7}$ & $1.00 \times 10^{-4}$ & $1.00 \times 10^{-2}$ & $6.88 \times 10^{-3}$ & $3.24 \times 10^{-11}$ & $7.19 \times 10^{-7}$ \\
\hline & & $\rho_{7}=14.5$ & $T_{9}=3.8$ & & $\begin{array}{c}\dot{Y}_{e \mathrm{DB}}(\mathrm{GT}) \\
2.43 \times 10^{-8}\end{array}$ & $\begin{array}{c}\dot{Y}_{e \mathrm{DB}}(\text { Auf }) \\
1.22 \times 10^{-6}\end{array}$ \\
\hline
\end{tabular}

Table 11

Beta Decay for the 16 More Relevant Nuclei at $Y_{e}=0.44$

\begin{tabular}{|c|c|c|c|c|c|c|}
\hline Nucleus & $\begin{array}{c}\lambda_{\mathrm{DB}}(\mathrm{GT}) \\
\left(\mathrm{s}^{-1}\right)\end{array}$ & $\begin{array}{c}\lambda_{\mathrm{DB}}(\mathrm{SM}) \\
\left(\mathrm{s}^{-1}\right)\end{array}$ & $\begin{array}{c}\lambda_{\mathrm{DB}}(\mathrm{Auf}) \\
\left(\mathrm{s}^{-1}\right)\end{array}$ & $\begin{array}{c}\lambda_{\mathrm{DB}}(\mathrm{FFN}) \\
\left(\mathrm{s}^{-1}\right)\end{array}$ & $\begin{array}{c}\left|\dot{Y}_{e \mathrm{DB}}(\mathrm{GT})\right| \\
\left(\mathrm{s}^{-1}\right)\end{array}$ & $\mid \begin{array}{c}\mid \dot{Y}_{e \mathrm{DB}}(\text { Auf }) \mid \\
\left(\mathrm{s}^{-1}\right)\end{array}$ \\
\hline$\overline{50} \mathrm{Sc}$ & $4.10 \times 10^{-2}$ & $1.10 \times 10^{-2}$ & $1.80 \times 10^{-1}$ & $2.80 \times 10^{-2}$ & $2.04 \times 10^{-7}$ & $2.08 \times 10^{-7}$ \\
\hline${ }^{54} \mathrm{~V}$ & $3.85 \times 10^{-2}$ & $\ldots$ & $1.90 \times 10^{-1}$ & $\ldots$ & $1.15 \times 10^{-7}$ & $2.04 \times 10^{-7}$ \\
\hline${ }^{58} \mathrm{Mn}$ & $5.80 \times 10^{-8}$ & $\ldots$ & $1.39 \times 10^{-1}$ & $\ldots$ & $8.70 \times 10^{-8}$ & $6.30 \times 10^{-7}$ \\
\hline${ }^{62} \mathrm{Co}$ & $1.64 \times 10^{-3}$ & $\ldots$ & $5.93 \times 10^{-2}$ & $\ldots$ & $5.03 \times 10^{-8}$ & $4.12 \times 10^{-7}$ \\
\hline${ }^{61} \mathrm{Fe}$ & $7.45 \times 10^{-4}$ & $1.70 \times 10^{-3}$ & $6.40 \times 10^{-2}$ & $\ldots$ & $3.45 \times 10^{-8}$ & $1.36 \times 10^{-6}$ \\
\hline${ }^{59} \mathrm{Mn}$ & $5.40 \times 10^{-3}$ & $\ldots$ & $1.58 \times 10^{-1}$ & $\ldots$ & $3.20 \times 10^{-8}$ & $2.89 \times 10^{-7}$ \\
\hline${ }^{53} \mathrm{~V}$ & $8.82 \times 10^{-5}$ & $\ldots$ & $2.98 \times 10^{-3}$ & $\ldots$ & $2.05 \times 10^{-8}$ & $2.63 \times 10^{-7}$ \\
\hline${ }^{63} \mathrm{Co}$ & $3.52 \times 10^{-4}$ & $1.50 \times 10^{-2}$ & $1.40 \times 10^{-2}$ & $\ldots$ & $2.00 \times 10^{-8}$ & $7.46 \times 10^{-7}$ \\
\hline${ }^{49} \mathrm{Ca}$ & $2.84 \times 10^{-2}$ & $\ldots$ & $4.18 \times 10^{-2}$ & $\ldots$ & $1.35 \times 10^{-8}$ & $7.27 \times 10^{-9}$ \\
\hline${ }^{53} \mathrm{Ti}$ & $1.95 \times 10^{-2}$ & $\ldots$ & $3.19 \times 10^{-2}$ & $\ldots$ & $1.24 \times 10^{-8}$ & $1.04 \times 10^{-8}$ \\
\hline${ }^{52} \mathrm{~V}$ & $1.08 \times 10^{-4}$ & $\ldots$ & $7.60 \times 10^{-3}$ & $\ldots$ & $1.10 \times 10^{-8}$ & $9.88 \times 10^{-8}$ \\
\hline${ }^{51} \mathrm{Sc}$ & $4.73 \times 10^{-2}$ & $\ldots$ & $1.32 \times 10^{-1}$ & $\ldots$ & $8.17 \times 10^{-9}$ & $6.16 \times 10^{-9}$ \\
\hline${ }^{56} \mathrm{Mn}$ & $4.58 \times 10^{-5}$ & $\ldots$ & $4.58 \times 10^{-3}$ & $\ldots$ & $5.89 \times 10^{-9}$ & $9.15 \times 10^{-8}$ \\
\hline${ }^{67} \mathrm{Ni}$ & $5.29 \times 10^{-4}$ & $\ldots$ & $1.20 \times 10^{-2}$ & $\ldots$ & $5.70 \times 10^{-9}$ & $1.05 \times 10^{-7}$ \\
\hline${ }^{55} \mathrm{Cr}$ & $1.12 \times 10^{-5}$ & $\ldots$ & $1.06 \times 10^{-3}$ & $\ldots$ & $5.57 \times 10^{-9}$ & $2.49 \times 10^{-7}$ \\
\hline \multirow[t]{2}{*}{${ }^{63} \mathrm{Fe}$} & $1.68 \times 10^{-1}$ & $\ldots$ & $\ldots$ & $\ldots$ & $5.33 \times 10^{-9}$ & $\ldots$ \\
\hline & & $\rho_{7}=33$ & $T_{9}=4.24$ & & $\begin{array}{c}\dot{Y}_{e \mathrm{DB}}(\mathrm{GT}) \\
6.39 \times 10^{-7}\end{array}$ & $\begin{array}{c}\dot{Y}_{e \mathrm{DB}}(\text { Auf }) \\
1.01 \times 10^{-5}\end{array}$ \\
\hline
\end{tabular}


Table 12

Beta Decay for the 19 More Relevant Nuclei at $Y_{e}=0.43$

\begin{tabular}{|c|c|c|c|c|c|c|}
\hline Nucleus & $\begin{array}{c}\lambda_{\mathrm{DB}}(\mathrm{GT}) \\
\left(\mathrm{s}^{-1}\right)\end{array}$ & $\begin{array}{c}\lambda_{\mathrm{DB}}(\mathrm{SM}) \\
\left(\mathrm{s}^{-1}\right)\end{array}$ & $\begin{array}{c}\lambda_{\mathrm{DB}} \text { (Auf) } \\
\left(\mathrm{s}^{-1}\right)\end{array}$ & $\begin{array}{c}\lambda_{\mathrm{DB}}(\mathrm{FFN}) \\
\left(\mathrm{s}^{-1}\right)\end{array}$ & $\begin{array}{c}\left|\dot{Y}_{e \mathrm{DB}}(\mathrm{GT})\right| \\
\left(\mathrm{s}^{-1}\right)\end{array}$ & $\begin{array}{c}\mid \dot{Y}_{e \mathrm{DB}}(\text { Auf }) \mid \\
\left(\mathrm{s}^{-1}\right)\end{array}$ \\
\hline${ }^{50} \mathrm{Sc}$ & $1.36 \times 10^{-2}$ & $\ldots$ & $1.10 \times 10^{-1}$ & $1.34 \times 10^{-2}$ & $1.90 \times 10^{-6}$ & $3.82 \times 10^{-6}$ \\
\hline${ }^{54} \mathrm{~V}$ & $1.29 \times 10^{-2}$ & $\ldots$ & $1.18 \times 10^{-1}$ & $5.14 \times 10^{-2}$ & $4.92 \times 10^{-7}$ & $1.82 \times 10^{-6}$ \\
\hline${ }^{49} \mathrm{Ca}$ & $6.00 \times 10^{-3}$ & $\ldots$ & $\ldots$ & $\ldots$ & $4.08 \times 10^{-7}$ & $\ldots$ \\
\hline${ }^{63} \mathrm{Fe}$ & $5.31 \times 10^{-2}$ & $\ldots$ & $7.18 \times 10^{-1}$ & $\ldots$ & $3.25 \times 10^{-7}$ & $5.81 \times 10^{-7}$ \\
\hline${ }^{51} \mathrm{Sc}$ & $1.35 \times 10^{-2}$ & $\ldots$ & $\ldots$ & $\ldots$ & $3.21 \times 10^{-7}$ & $\ldots$ \\
\hline${ }^{53} \mathrm{Ti}$ & $3.70 \times 10^{-3}$ & $\ldots$ & $\ldots$ & $\ldots$ & $1.40 \times 10^{-7}$ & $\ldots$ \\
\hline${ }^{58} \mathrm{Mn}$ & $1.52 \times 10^{-3}$ & $\ldots$ & $1.61 \times 10^{-1}$ & $1.22 \times 10^{-1}$ & $1.06 \times 10^{-7}$ & $3.98 \times 10^{-6}$ \\
\hline${ }^{59} \mathrm{Mn}$ & $1.05 \times 10^{-3}$ & $3.50 \times 10^{-1}$ & $3.50 \times 10^{-1}$ & $1.00 \times 10^{+0}$ & $9.02 \times 10^{-8}$ & $1.04 \times 10^{-5}$ \\
\hline${ }^{61} \mathrm{Fe}$ & $8.52 \times 10^{-5}$ & $\ldots$ & $1.25 \times 10^{-1}$ & $\ldots$ & $2.99 \times 10^{-8}$ & $2.16 \times 10^{-5}$ \\
\hline${ }^{60} \mathrm{Mn}$ & $1.83 \times 10^{-2}$ & $\ldots$ & $\ldots$ & $\ldots$ & $2.37 \times 10^{-8}$ & $\ldots$ \\
\hline${ }^{67} \mathrm{Ni}$ & $4.80 \times 10^{-5}$ & $\ldots$ & $1.71 \times 10^{-2}$ & $\ldots$ & $2.08 \times 10^{-8}$ & $5.38 \times 10^{-6}$ \\
\hline${ }^{62} \mathrm{Co}$ & $2.98 \times 10^{-4}$ & $\ldots$ & $7.16 \times 10^{-2}$ & $\ldots$ & $1.81 \times 10^{-8}$ & $1.18 \times 10^{-6}$ \\
\hline${ }^{63} \mathrm{Co}$ & $3.41 \times 10^{-5}$ & $\ldots$ & $2.01 \times 10^{-2}$ & $\ldots$ & $9.48 \times 10^{-9}$ & $5.40 \times 10^{-6}$ \\
\hline${ }^{50} \mathrm{Ca}$ & $2.12 \times 10^{-3}$ & $\ldots$ & $\ldots$ & $\ldots$ & $7.61 \times 10^{-9}$ & $\ldots$ \\
\hline${ }^{53} \mathrm{~V}$ & $7.84 \times 10^{-6}$ & $\ldots$ & $\ldots$ & $\ldots$ & $4.05 \times 10^{-9}$ & $\ldots$ \\
\hline${ }^{52} \mathrm{~V}$ & $1.17 \times 10^{-5}$ & $\ldots$ & $\ldots$ & $\ldots$ & $9.83 \times 10^{-10}$ & $\ldots$ \\
\hline${ }^{51} \mathrm{Ti}$ & $1.06 \times 10^{-6}$ & $\ldots$ & $2.13 \times 10^{-3}$ & $1.13 \times 10^{-3}$ & $8.01 \times 10^{-10}$ & $1.22 \times 10^{-6}$ \\
\hline${ }^{55} \mathrm{Cr}$ & $9.08 \times 10^{-7}$ & $\ldots$ & $3.31 \times 10^{-3}$ & $6.05 \times 10^{-3}$ & $5.67 \times 10^{-10}$ & $1.07 \times 10^{-6}$ \\
\hline \multirow[t]{2}{*}{${ }^{62} \mathrm{Fe}$} & $1.11 \times 10^{-6}$ & $\ldots$ & $6.38 \times 10^{-2}$ & $\ldots$ & $3.33 \times 10^{-10}$ & $8.63 \times 10^{-6}$ \\
\hline & & $\rho_{7}=106$ & $T_{9}=4.93$ & & $\begin{array}{c}\dot{Y}_{e \mathrm{DB}}(\mathrm{GT}) \\
3.90 \times 10^{-6}\end{array}$ & $\begin{array}{c}\dot{Y}_{e \mathrm{DB}}(\text { Auf }) \\
9.57 \times 10^{-5}\end{array}$ \\
\hline
\end{tabular}

Table 13

Beta Decay for the 12 More Relevant Nuclei at $Y_{e}=0.425$

\begin{tabular}{|c|c|c|c|c|c|c|}
\hline Nucleus & $\begin{array}{c}\lambda_{\mathrm{DB}}(\mathrm{GT}) \\
\left(\mathrm{s}^{-1}\right)\end{array}$ & $\begin{array}{c}\lambda_{\mathrm{DB}}(\mathrm{SM}) \\
\left(\mathrm{s}^{-1}\right)\end{array}$ & $\begin{array}{c}\lambda_{\mathrm{DB}}(\text { Auf }) \\
\left(\mathrm{s}^{-1}\right)\end{array}$ & $\begin{array}{c}\lambda_{\mathrm{DB}}(\mathrm{FFN}) \\
\left(\mathrm{s}^{-1}\right)\end{array}$ & $\begin{array}{c}\left|\dot{Y}_{e \mathrm{DB}}(\mathrm{GT})\right| \\
\left(\mathrm{s}^{-1}\right)\end{array}$ & $\begin{array}{c}\mid \dot{Y}_{e \mathrm{DB}}(\text { Auf }) \mid \\
\left(\mathrm{s}^{-1}\right)\end{array}$ \\
\hline$\overline{{ }^{50} \mathrm{Sc}}$ & $4.19 \times 10^{-3}$ & $\ldots$ & $5.54 \times 10^{-2}$ & $\ldots$ & $1.44 \times 10^{-6}$ & $5.16 \times 10^{-6}$ \\
\hline${ }^{63} \mathrm{Fe}$ & $1.47 \times 10^{-2}$ & $\ldots$ & $3.54 \times 10^{-1}$ & $\ldots$ & $4.27 \times 10^{-7}$ & $1.61 \times 10^{-6}$ \\
\hline${ }^{51} \mathrm{Sc}$ & $3.64 \times 10^{-3}$ & $\ldots$ & $3.44 \times 10^{-2}$ & $\ldots$ & $8.66 \times 10^{-7}$ & $1.02 \times 10^{-6}$ \\
\hline${ }^{49} \mathrm{Ca}$ & $1.22 \times 10^{-3}$ & $\ldots$ & $9.31 \times 10^{-3}$ & $\ldots$ & $3.91 \times 10^{-7}$ & $1.14 \times 10^{-6}$ \\
\hline${ }^{54} \mathrm{~V}$ & $4.05 \times 10^{-3}$ & $\ldots$ & $6.08 \times 10^{-2}$ & $\ldots$ & $2.72 \times 10^{-7}$ & $1.87 \times 10^{-6}$ \\
\hline${ }^{53} \mathrm{Ti}$ & $7.14 \times 10^{-4}$ & $\ldots$ & $1.47 \times 10^{-2}$ & $\ldots$ & $8.66 \times 10^{-8}$ & $1.07 \times 10^{-6}$ \\
\hline${ }^{60} \mathrm{Mn}$ & $7.78 \times 10^{-3}$ & $\ldots$ & $3.38 \times 10^{-1}$ & $\ldots$ & $3.92 \times 10^{-8}$ & $1.22 \times 10^{-6}$ \\
\hline${ }^{59} \mathrm{Mn}$ & $2.06 \times 10^{-4}$ & $2.10 \times 10^{-2}$ & $1.40 \times 10^{-1}$ & $7.20 \times 10^{-1}$ & $3.03 \times 10^{-8}$ & $7.93 \times 10^{-6}$ \\
\hline${ }^{58} \mathrm{Mn}$ & $3.75 \times 10^{-4}$ & $\ldots$ & $7.25 \times 10^{-2}$ & $\ldots$ & $2.96 \times 10^{-8}$ & $2.35 \times 10^{-6}$ \\
\hline${ }^{50} \mathrm{Ca}$ & $3.79 \times 10^{-4}$ & $\ldots$ & $\ldots$ & $\ldots$ & $1.36 \times 10^{-8}$ & $\ldots$ \\
\hline${ }^{67} \mathrm{Ni}$ & $7.88 \times 10^{-6}$ & $\ldots$ & $4.02 \times 10^{-3}$ & $\ldots$ & $7.85 \times 10^{-9}$ & $2.85 \times 10^{-6}$ \\
\hline \multirow[t]{2}{*}{${ }^{59} \mathrm{Fe}$} & $1.46 \times 10^{-5}$ & $\ldots$ & $\ldots$ & $\ldots$ & $6.48 \times 10^{-9}$ & $\ldots$ \\
\hline & & $\rho_{7}=220$ & $T_{9}=5.39$ & & $\begin{array}{c}\dot{Y}_{e \mathrm{DB}}(\mathrm{GT}) \\
3.16 \times 10^{-6}\end{array}$ & $\begin{array}{c}\dot{Y}_{e \mathrm{DB}}(\text { Auf }) \\
6.87 \times 10^{-5}\end{array}$ \\
\hline
\end{tabular}

calculates satisfactory rates for the values of $Y_{e}$ in which the nucleus contributes with its rate for the biggest value of $\dot{Y}_{e}$, related with other nuclei in the same point. Figure 7 shows an intersection between two curves of SM and GT in the same value of $Y_{e}$, where ${ }^{56} \mathrm{Co}$ and ${ }^{58} \mathrm{Co}$ present the higher magnitude order on its derivative $\dot{Y}_{e}$. For example, look at ${ }^{56} \mathrm{Co}$ in Table 2 and ${ }^{58} \mathrm{Co}$ in Table 3 , and observe that they do not appear at the top of Tables 4-7. This effect is because they show a temporal derivative $\dot{Y}_{e}$, which is orders of magnitude smaller than those of the nuclei with more contribution to the EC at these points of the stellar trajectory.

We also perform this analysis for the BD rates of ${ }^{56} \mathrm{Mn}$, because we observed that this nucleus gives a relevant contribution to the $\dot{Y}_{e}$ value of Auf and also for the GT in several points of the stellar trajectory (see Tables 8 and 9). As is shown in Figure 8, our rates calculated for ${ }^{56} \mathrm{Mn}$ are at least one magnitude order below the other three model's results. This is a general tendency of the rates evaluated within the GT, which gives lower or similar values to the other compared models for the majority of the analyzed species. For EC, only two rates are bigger than those calculated with FFN and SM. For BD, we observe that only one rate is above the value found by Auf. Consequently, the sum of the temporal derivatives of $Y_{e}$ are higher than those calculated by Auf in all the points. This behavior could be related to the fact that we are only considering here the nuclear transition from the ground state, as it was proposed in the original version of the GT.

As shown in Tables 1-7, any of the more important values of $\dot{Y}_{e}$ for EC calculated with the GT are bigger than those of Auf, being sometimes of the same magnitude order. Moreover, the nuclei that exhibit the higher values of $\dot{Y}_{e}$ in a specific point of $Y_{e}$ for GT are not always the same ones obtained by Auf. For BD, we observe the same behavior. It is important to note that Table 8 indicates ${ }^{56} \mathrm{Mn}$ is the nucleus with a higher value of $\dot{Y}_{e}$. On the other hand, in Table 19 from Aufderheide et al. (1994b) ${ }^{56} \mathrm{Co}$ is the nucleus with higher $\dot{Y}_{e}$, with its value being $1.51 \times 10^{-13}$ (i.e., four orders of magnitude higher than those of $\left.{ }^{56} \mathrm{Mn}\right)$. However, for this nucleus, the GT model adopted 

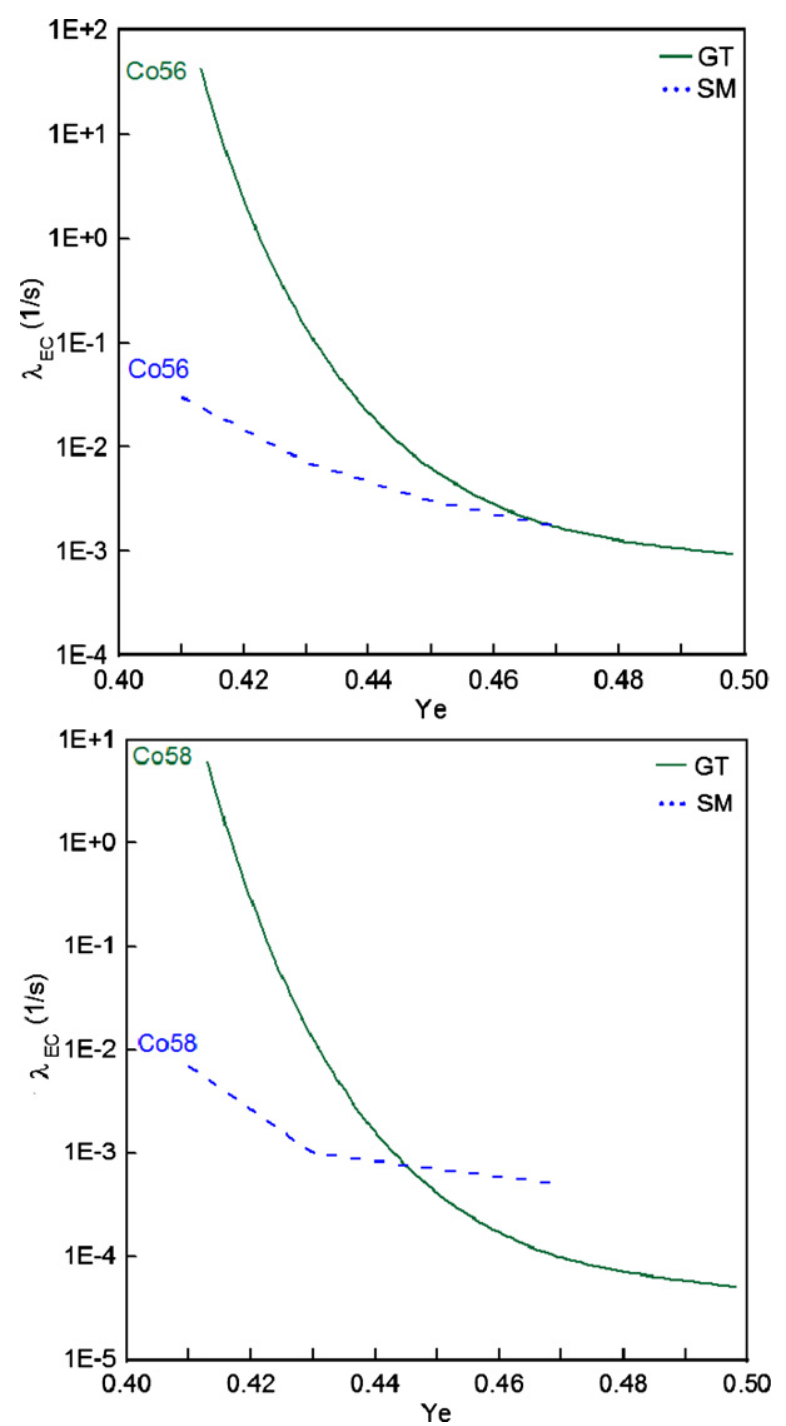

Figure 7. Electron capture rates for ${ }^{56} \mathrm{Co}$ and ${ }^{58} \mathrm{Co}$.

(A color version of this figure is available in the online journal.)

in this work can not to evaluate its rate, because we are only considering the transition from the ground state and the $Q$-value is negative.

Another test of our calculation can be performed. A recent systematic evaluation of the ability of theoretical models to reproduce experimental Gamow-Teller transition strength distributions measured using the $(n, p)$-type charge-exchange reactions at intermediate beam energies was performed by Cole et al. (2012). We compare our EC rates in the GT with the results presented in that work. There, the EC rates were evaluated in astrophysical conditions of temperature and density as functions of $Y_{e}$, which are not at all compatible with our stellar trajectories shown in Equation (23). Even so, we observe that with $T_{9}=5.98$ (which corresponds to take $Y_{e}=0.423$ in Equation (23)) the value $\rho Y_{e}=10^{9} \mathrm{~g} \mathrm{~cm}^{-3}$ is compatible with our stellar trajectory and allows us to compare our results with those shown in Figures 6, 8, 12, 14, 16, 18, and 20 from Cole et al. (2012). In Figure 9 we compare $\log \left(\lambda_{\mathrm{EC}}\right)$ versus the mass number for the ${ }^{50} \mathrm{~V},{ }^{51} \mathrm{~V},{ }^{55} \mathrm{Mn},{ }^{56} \mathrm{Fe},{ }^{58} \mathrm{Ni},{ }^{59} \mathrm{Co}$, and ${ }^{60} \mathrm{Ni}$ nuclei with the available ${ }^{Z} A(n, p),{ }^{Z} A\left(d, 2^{H} e\right)$, or ${ }^{Z} A(n, p) T=3$ experimental data, and also with other theoretical evaluations, such as QRPA and SM (KB3G and GXPF1) from Cole et al. (2012). We note that the EC rates of GT, similar to the KB3G

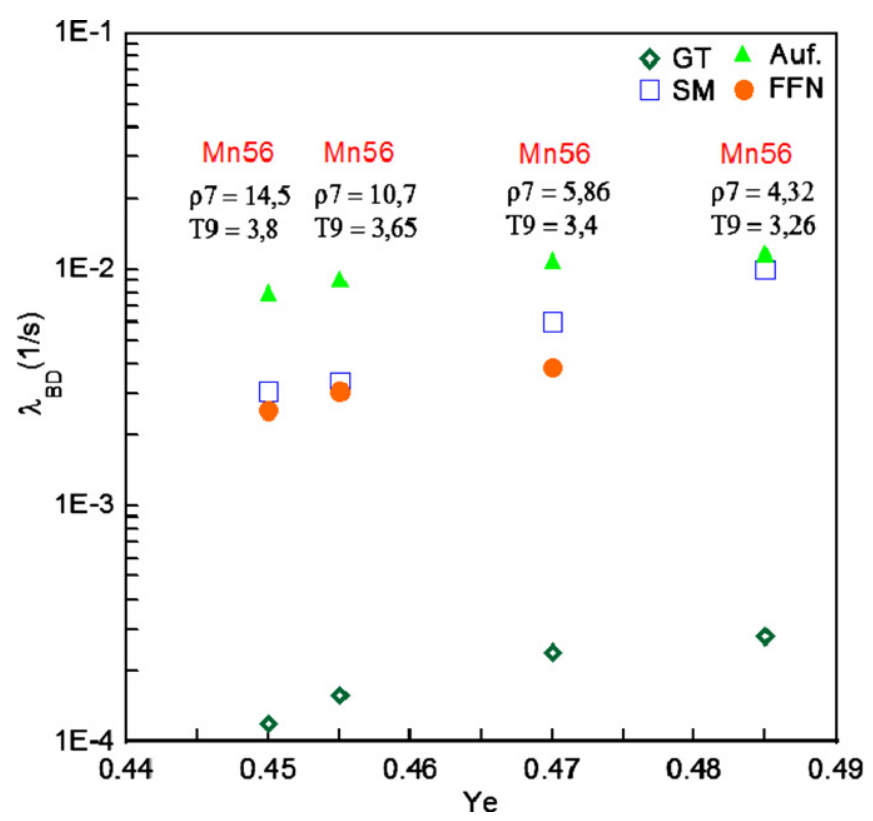

Figure 8. Beta decay rates for ${ }^{56} \mathrm{Mn}$ calculated from Equation (29). SM values from Martínez-Pinedo et al. (2000), FFN and Auf values from Aufderheide et al. (1994b).

(A color version of this figure is available in the online journal.)

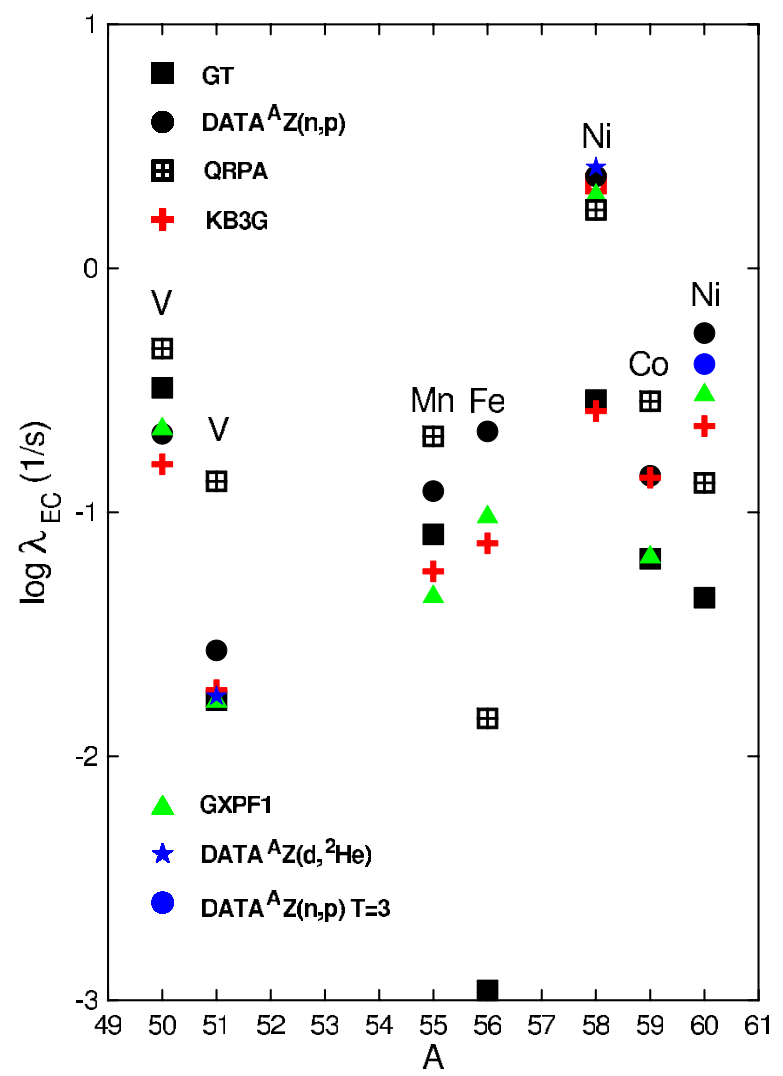

Figure 9. Comparison of GT results with available ${ }^{Z} A(n, p),{ }^{Z} A\left(d, 2^{H} e\right)$ or ${ }^{Z} A(n, p) T=3$ experimental data, and also with other theoretical QRPA and SM (KB3G and GXPF1) results from Cole et al. (2012), in similar stellar conditions.

(A color version of this figure is available in the online journal.)

and GXPF1a interactions, qualitatively reproduce experimental Gamow-Teller strength distributions of the seven stable isotopes compared, better than those obtained with microscopic QRPA 


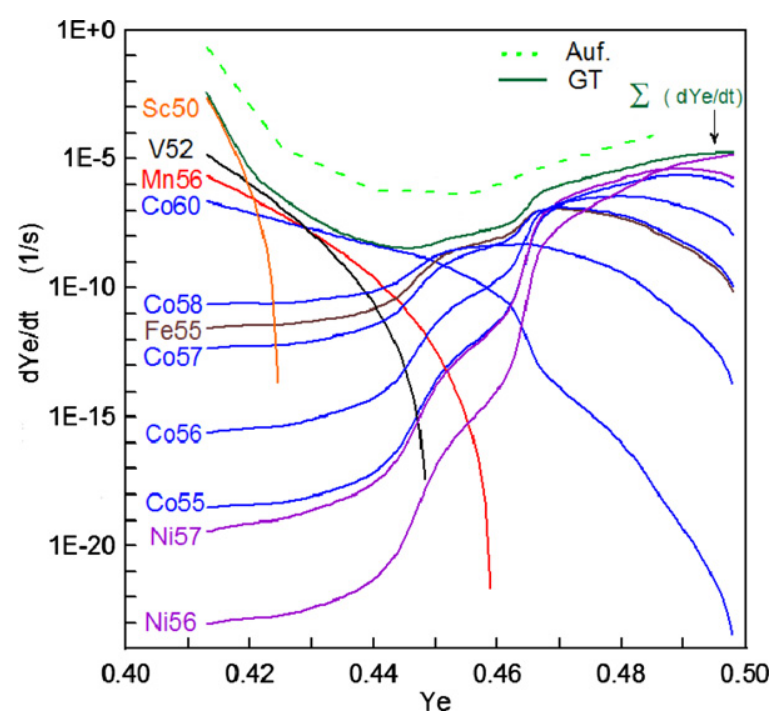

Figure 10. Temporal derivative $\dot{Y}_{e}$ for EC.

(A color version of this figure is available in the online journal.)

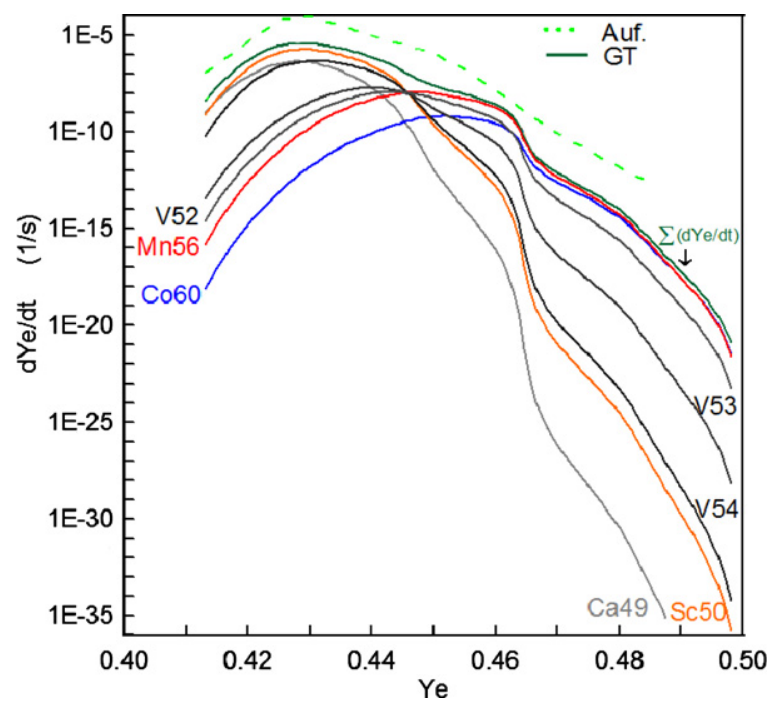

Figure 11. Temporal derivative $\dot{Y}_{e}$ for BD.

(A color version of this figure is available in the online journal.)

calculations. GT only observes a larger deviation from the data, $\mathrm{SM}$, and the QRPA results for ${ }^{56} \mathrm{Fe}$ and ${ }^{60} \mathrm{Ni}$.

We also analyzed the behavior of the temporal derivative $\dot{Y}_{e}$. In Figure 10 we plotted the 11 EC nuclei with higher $\dot{Y}_{e}$ values. For completeness, the solid lines represent the sum of $\dot{Y}_{e}$ and the dotted line represent $\dot{Y}_{e}$ values calculated by Auf for the eight selected points of the stellar trajectory. Figure 11 shows the same results for seven nuclei with higher value of $\dot{Y}_{e}$ for BD. Figure 12 exhibits the sum of $\dot{Y}_{e}$ values for EC and BD, with solid lines representing the GT results, the dashed lines represent those of Auf from Aufderheide et al. (1994b), and the short dashed lines represent those calculated with the SM from Martínez-Pinedo et al. (2000). In this figure, the lines perpendicular to the $Y_{e}$ axis represent the intervals where BD results exceed the EC ones when they are calculated within the mentioned models.

From Figures $10-12$ we observe that for $Y_{e} \approx 0.50$ the EC dominates because the $\dot{Y}_{e \mathrm{EC}}$ values for ${ }^{56} \mathrm{Ni},{ }^{57} \mathrm{Ni}$, and ${ }^{55} \mathrm{Co}$ are greater than the $\dot{Y}_{e \mathrm{BD}}$ for ${ }^{56} \mathrm{Mn}$ and ${ }^{60} \mathrm{Co}$, which have the domain of BD in this point of the stellar trajectory. When $Y_{e}$ diminishes, $\dot{Y}_{e \mathrm{EC}}$ decreases and $\dot{Y}_{e \mathrm{BD}}$ increases. More

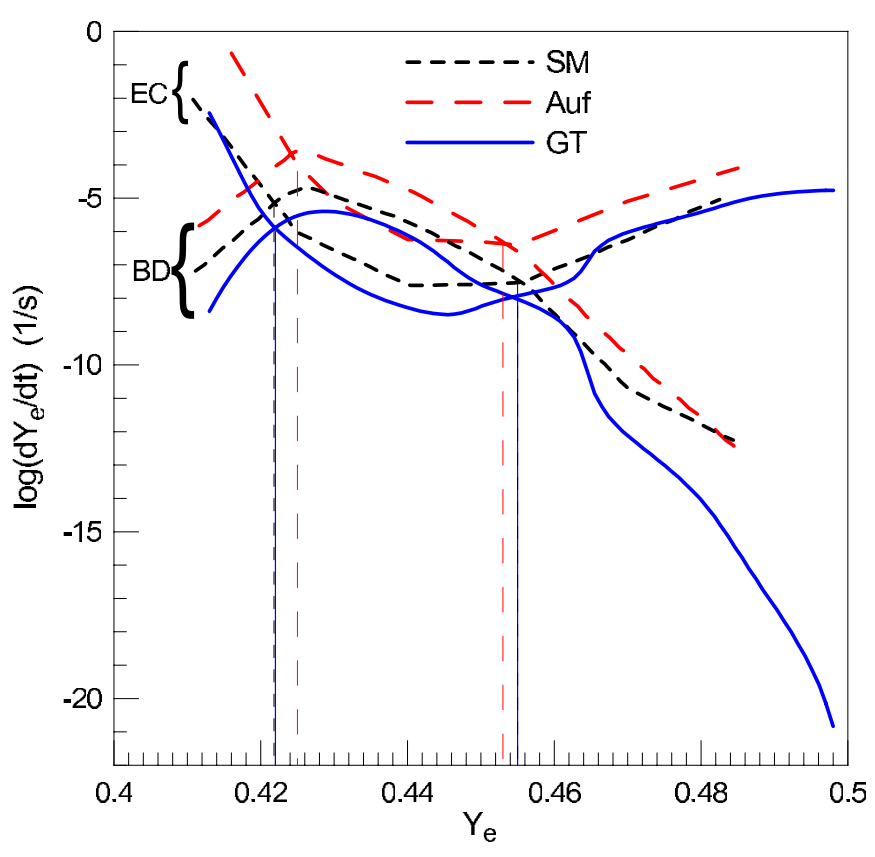

Figure 12. Temporal derivative $\dot{Y}_{e}$.

(A color version of this figure is available in the online journal.)

specifically, at $Y_{e}=0.455$ the $\dot{Y}_{e \mathrm{EC}}$ contributions are greater for ${ }^{57} \mathrm{Co},{ }^{55} \mathrm{Fe}$, and ${ }^{58} \mathrm{Co}$, which, when summed with the others in the same point, give a total $\dot{Y}_{e \mathrm{EC}}$ value smaller than the corresponding one to the BD. The total $\dot{Y}_{e \text { BD }}$ has increased, mainly because of the contribution of ${ }^{56} \mathrm{Mn}$ and ${ }^{52} \mathrm{~V}$, and the diminution in the $\dot{Y}_{e \text { EC }}$ value remains up to $Y_{e} \approx 0.44$. From this point, where the temperature is around $4.24 \times 10^{9} \mathrm{~K}$ and the density $3.30 \times 10^{8} \mathrm{~g} \mathrm{~cm}^{-3}$, the EC grows with the main contribution coming from ${ }^{60} \mathrm{Co}$, but still being smaller than the BD. The last one, besides being higher, continues to increase, having ${ }^{50} \mathrm{Sc}$ and ${ }^{54} \mathrm{~V}$ as dominant nuclei at this point. This behavior is observed up to $Y_{e} \approx 0.43$, where the temperature is around $4.93 \times 10^{9} \mathrm{~K}$ and the density $1.06 \times 10^{9} \mathrm{~g} \mathrm{~cm}^{-3}$. At this point, the total $\dot{Y}_{e \mathrm{DB}}$ has a contribution coming mainly from ${ }^{50} \mathrm{Sc}$, where the BD starts to diminish, but still exceeds the EC, which continues growing up with a total $\dot{Y}_{e \text { EC }}$ due to ${ }^{57} \mathrm{Co},{ }^{55} \mathrm{Fe}$, and ${ }^{58} \mathrm{Co}$. This situation is extended up to $Y_{e}=0.422$, where the $\mathrm{EC}$ returns to be dominant over $\mathrm{BD}$, with a temperature $6.00 \times 10^{9} \mathrm{~K}$ and a density $3.26 \times 10^{10} \mathrm{~g} \mathrm{~cm}^{-3}$. At this point, ${ }^{50} \mathrm{Sc}$ is still the nucleus with higher contribution to $\dot{Y}_{e \mathrm{DB}}$, whereas $\dot{Y}_{e \mathrm{EC}}$ is dominated by ${ }^{52} \mathrm{~V}$.

We have observed that the increase of the density within a short interval of $Y_{e}$ is the main reason for having EC rates that show a common increase for the final values of $Y_{e}$, and a $\dot{Y}_{e}$ that exhibits a quick increase from $Y_{e}=0.41$ to $Y_{e} \approx 0.44$ (e.g., Figures 2, 3, and 10). BD in the same interval present decreasing rates and $\dot{Y}_{e}$ (e.g., Figures 4, 5, and 11).

\section{FINAL REMARKS}

We evaluated the $\mathrm{BD}$ and $\mathrm{EC}$ rates within the original version of the GT, which only considers transitions from the ground state in the parent nucleus. Our calculations included a set of 63 nuclear species that are of relevance in presupernova environments. We tested the efficiency of our model by comparing our results with laboratory experimental data, obtaining satisfactory agreements.

We analyzed the rates in stellar conditions considering the effects of the density, temperature, and nuclear partition function. 
We used the nuclear partition function defined in Equation (8) from Dimarco et al. (2002). For density and temperature, we adopted the same parameterization used by Auf (Aufderheide et al. 1994b).

In order to study the influence of our GT rates on quantities of astrophysical interest, we calculated the temporal derivative of the electron fraction, $\dot{Y}_{e}$, using the isotopic abundances from Dimarco et al. (2002). We compared our rates with those obtained using the models of FFN (Fuller et al. 1982a), SM (Martínez-Pinedo et al. 2000), and Auf (Aufderheide et al. 1994b).

The rates evaluated within our GT model are smaller than those obtained from other models and we also found an interval where BD exceeds EC. This is a very important characteristic in the pre-supernova phase of massive stars already pointed out by Aufderheide et al. (1994b, 1996) and recently by MartínezPinedo et al. (2000). In Figure 12 we show a comparison between the results for $\log \left(d Y_{e} / d t\right)$ (in units of $1 \mathrm{~s}^{-1}$ ) versus $Y_{e}$ obtained within our GT and those calculated with the SM from MartínezPinedo et al. (2000) and the Auf model from Aufderheide et al. (1994b, 1996). Our GT results show that the interval where $\dot{Y}_{e}$ for $\mathrm{BD}$ is higher than those of EC goes from $\approx 0.422-0.455$. This is $\sim 18 \%$ greater than the corresponding interval proposed by Auf, which goes from $\approx 0.425-0.453$, and so closed to the $\mathrm{SM}$ results (the quotient is less than $1 \%$ ). We emphasize that the GT enforces the tendency of an existing interval, higher than those proposed by Auf, where BD exceeds EC, in the same way as the SM results predict.

The increment of this interval could have important consequences over the core collapse, because weak processes determine the amount of neutrinos emitted and consequently affect the temperature, the lepton fraction, and entropy profiles of the core. From Figure 12, we pointed out that the interval where the BD exceeds EC is very similar for the GT and SM results. The main feature is that both BD and EC are slower in the GT than in the SM. This means that a smaller quantity of energy will be transported by neutrinos outside the core, leading to a hotter core in the pre-supernova stage than one predicted by SM calculations. On the other hand, as both rates have approximately the same temperature dependence of Martínez-Pinedo et al. (2000), the GT also predicts that the used stellar trajectory is consistent. The fact that both GT rates are below the SM ones in the above interval could have important consequences over the core collapse. Some of these consequences were studied by Aufderheide et al. (1994a), where the authors claimed that the competition between $\mathrm{BD}$ and EC leads to cooler cores and larger $Y_{e}$ values at the formation of the homologous core.

In summary, the contributions that the GT can to bring along this research line are relevant. These calculations are well supported because we have calculated decay rates that are in good agreement with other models. We stress the fact that the GT is simple from the computational point of view and useful for systematic calculations that involve a large number of nuclei. Thus, it is also an alternative for EC calculations along the collapse because, as mentioned by Juodagalvis et al. (2010), in this stage of stellar evolution the number of nuclei can reach almost 2700 species. However, it is important to note that it will be important to perform an advanced study using the GT and/or its actualization, like the Semi-Gross theory, for transitions not only from the ground state but also considering excited states in the parent nucleus and to include the first forbidden transitions. Within this context, higher decay rates could be expected with the GT for two reasons: (1) in the present calculations, the contribution to the excited states was not included and (2) the contribution of BD of some of the 34 nuclei are not employed here because having $Q<0$ as for example ${ }^{56} \mathrm{Co}$ and ${ }^{54} \mathrm{Mn}$, which are considered to be important by Aufderheide et al. (1994b). These considerations could increase the value of $\dot{Y}_{e}$ for $\mathrm{BD}$, further increasing its excess interval in comparison with EC. This analysis will be performed in future studies.

\section{REFERENCES}

Alford, W. P., Helmer, R. L., Abegg, R., et al. 1990, NuPhA, 514, 49 Arnet, W. D. 1967, CaJPh, 45, 1621

Aufderheide, M. B., Bloom, S. D., Mathews, G. J., \& Resler, D. A. 1996, PhRvC, 53,3139

Aufderheide, M. B., Brown, G. E., Kuo, T. T. S., Stout, D. B., \& Vogel, P. 1990, ApJ, 362, 241

Aufderheide, M. B., Fushiki, I., Fuller, G. M., \& Weaver, T. A. 1994a, ApJ, 424, 257

Aufderheide, M. B., Fushiki, I., Woosley, S. E., \& Hartmann, D. H. 1994b, ApJS, 91,389

Bahcall, J. N. 1964, ApJ, 139, 318

Borzov, I. N., \& Goriely, S. 2000, PhRvC, 62, 035501

Caurier, E., Langanke, K., Martínez-Pinedo, G., \& Nowacki, F. 1999, NuPhA, 653,439

Cole, A. L., Anderson, T. S., Zegers, R. G. T., et al. 2012, PhRvC, 86, 015809

Colgate, S. A., \& White, R. H. 1966, ApJ, 143, 626

Dean, D. J., Langanke, K., Chatterjee, L., Radha, P. B., \& Strayer, M. R. 1998, PhRvC, 58, 536

Dimarco, A. J., Barbero, C., Dias, H., et al. 2002, JPhG, 28, 121

Egawa, Y., Yokoi, K., \& Yamada, M. 1975, PThPh, 54, 1339

El-Kateb, S., Jackson, K. P., Alford, W. P., et al. 1994, PhRvC, 49, 3128

Feenberg, E., \& Trigg, G. 1950, RvMP, 22, 399

Fowler, W. A., \& Holey, F. 1964, ApJS, 09, 20

Fuller, G. M., Fowler, W. A., \& Newman, M. J. 1980, ApJS, 42, 447

Fuller, G. M., Fowler, W. A., \& Newman, M. J. 1982a, ApJ, 252, 715

Fuller, G. M., Fowler, W. A., \& Newman, M. J. 1982b, ApJS, 48, 279

Fuller, G. M., Fowler, W. A., \& Newman, M. J. 1985, ApJ, 293, 1

Goriely, S., \& Khan, E. 2002, NuPhA, 706, 217

Heger, A., Langanke, K., Martínez-Pinedo, G., \& Woosley, S. E. 2000, PhRvL, 86,1678

Heger, A., Woosley, S. E., Martínez-Pinedo, G., \& Langanke, K. 2001, ApJ, 560,307

Itoh, N., \& Kohyama, Y. 1977, NuPhA, 306, 527

Juodagalvis, A., Langanke, K., Hix, W. R., Martínez-Pinedo, G., \& Sampaio, J. M. 2010, NuPhA, 848, 454

Kar, K., Ray, A., \& Sarkar, S. 1994, ApJ, 434, 662

Kondoh, T., Tachibana, T., \& Yamada, M. 1985, PThPh, 74, 708

Koyama, S., Takahashi, K., \& Yamada, M. 1970, PThPh, 44, 663

Langanke, K. 1999, PhRvL, 83, 4502

Langanke, K., \& Martínez-Pinedo, G. 1999, PhLB, 453, 187

Langanke, K., \& Martínez-Pinedo, G. 2001, ADNDT, 79, 1

Letter of Nuclide 2013, available at: www-nds.iaea.org/relnsd/vchart/index.html Martínez-Pinedo, G. 2001, NuPhA, 688, 357

Martínez-Pinedo, G., Langanke, K., \& Dean, D. J. 2000, ApJS, 126, 493

Mazurek, T. J., Cameron, A. G. W., \& Truran, J. 1974, Ap\&SS, 27, 261

Nakata, H., Tachibana, T., \& Yamada, M. 1997, NuPhA, 625, 521

Nakayama, K., Galeão, A. P., \& Krmpotić, F. 1982, PhLB, 114, 217

Niu, Y. F., Paar, N., Vretenar, D., \& Meng, J. 2011, PhRvC, 83, 045807

Petterson, V. L., \& Bahcall, J. N. 1963, ApJ, 138, 437

Qian, Y. Z., Haxton, W. C., Langanke, K., \& Vogel, P. 1997, PhRvC, 55, 1532

Ronnquist, T. F., Condé, H., \& Olsson, N. 1993, NuPhA, 563, 225

Rose, M. E. (ed.) 1961, Relativistic Electron Theory (New York: Wiley), chap. V

Samana, A. R., Barbero, C. A., Duarte, B., Dimarco, A. J., \& Krmpotić, F. 2008, NJPh, 10, 033007

Sampaio, J. M., Langanke, K., Martínez-Pinedo, G., Kolbe, E., \& Dean, D. J. 2003, NuPhA, 718, 440

Tachibana, T., Yamada, M., \& Yoshida, Y. 1990, PThPh, 84, 641

Takahashi, K., \& Yamada, M. 1969, PThPh, 41, 1470

Türler, M. 2006, CERN Cour., 46, 1

Vetterly, M. C., Häusser, O., \& Abegg, R. 1989, PhRvC, 40, 559

Weaver, M. C., Zimmerman, G. B., \& Woosley, S. E. 1978, ApJ, 225, 1021

Williams, A. L., Alford, W. P., Brash, E., et al. 1995, PhRvC, 51, 1144 